\title{
Grain scale simulation of local deformation behavior
}

\author{
Tung Van Phan ${ }^{1,2}$
}

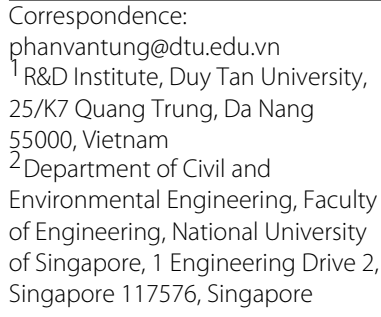

\begin{abstract}
In this work, a full-field finite element simulation of a heterogeneous DC04 steel microstructure identified from two-dimensional (2D) electron backscatter diffraction (EBSD) data is performed under a macroscopic tensile deformation. After discretization procedure by finite elements, the EBSD microstructure is subjected to homogeneous displacement boundary conditions approximately describing a large strain uniaxial tensile test. A crystal plasticity model applied on integration points of FE method is used to simulate the deformation behavior and the grain orientation evolution. The simulated grain orientation fields are compared to experimental measurements of the specimen after the tensile test at different deformation levels.
\end{abstract}

\section{Background}

Most of the metals used in industrial processes are polycrystalline materials. They are aggregates of approximately single crystals in grains with different crystal orientations. The anisotropic plasticity of polycrystalline materials is mainly caused by non-uniform distributions of crystal orientations [1]. Therefore, the analysis of the crystallographic texture, i.e., preferred crystal orientations, plays an important role when investigating the macroscopic material behavior. Crystallographic texture data allow for the characterization and prediction of the anisotropic plasticity in heterogeneous materials by using statistical models. Some representative studies of different polycrystalline materials with focus on the texture evolution are given, e.g., by [2-10]. In the intensive increase of computer powerful tool, modelling and simulation have contributed to predict the mechanical behavior and to facilitate the material design with particular properties. This issue often requires a computational framework based on the linking between the constitutive law describing physical phenomenon and the experimental information at the mesoscopic and microscopic level. The experimental electron backscatter diffraction (EBSD) technique, known as scanning electron microscope (SEM)-based technique, has become a major tool in measuring crystal orientations from a polycrystalline structure. A common application is the use of orientation data at every Gauss integration point in finite element (FE) simulations of crystal plasticity models. Hence, a grain scale simulation of the polycrystalline structure could be performed to describe the grain orientation evolution.

In this paper, a heat-treated DC04 steel microstructure identified from two-dimensional (2D) EBSD data is considered. A grain structure model is constructed based on the DC04 
steel microstructure identified by a Matlab toolbox MTEX. After discretized by finite elements with one element over thickness, a grain scale simulation of the polycrystalline DC04 steel sample cut from a tensile specimen is performed within the finite strain crystal plasticity framework. The crystal plasticity model accounting for specified grain orientations is applied at Gauss integration points of finite elements of the corresponding grains simultaneously. The experimentally observed local grain orientations and reorientations in the polycrystalline sample will be predicted and evaluated at different deformation states. Subsequently, the simulated grain reorientation fields are compared to experimental measurements of the DC04 sample after the tensile test at different deformation levels.

Notation. Throughout the text, a second-order tensor and a fourth-order tensor are $\boldsymbol{A}=A_{i j} \boldsymbol{e}_{i} \otimes \boldsymbol{e}_{j}$ and $\mathbb{A}=A_{i j k l} \boldsymbol{e}_{i} \otimes \boldsymbol{e}_{j} \otimes \boldsymbol{e}_{k} \otimes \boldsymbol{e}_{l}$, respectively, where $\left\{\boldsymbol{e}_{i}\right\}$ represents an orthonormal basis of the three-dimensional (3D) Euclidean space. Symmetric and traceless tensors are designated by a prime, e.g., $\boldsymbol{A}^{\prime}$. The set of proper orthogonal second-order tensors is specified by $S O(3)$. The scalar product, the dyadic product, and the Frobenius norm are denoted by $\boldsymbol{A} \cdot \boldsymbol{B}=\operatorname{tr}\left(\boldsymbol{A}^{\top} \boldsymbol{B}\right)=\operatorname{tr}\left(\boldsymbol{A} \boldsymbol{B}^{\top}\right), \boldsymbol{A} \otimes \boldsymbol{B}$, and $\|\boldsymbol{A}\|=(\boldsymbol{A} \cdot \boldsymbol{A})^{1 / 2}$, respectively. Here, $\operatorname{tr}(\cdot)$ represents the trace of a second-order tensor. A linear mapping of second-order tensors is written as $\boldsymbol{A}=\mathbb{C}[\boldsymbol{B}]$.

\section{Methods}

The first subsection introduces the constitutive equations of a crystal plasticity material model applied at Gauss integration points of FE model. The constitutive model is described via an ABAQUS user subroutine UMAT allowing to define the mechanical behavior of material. The EBSD measurement technique to obtain the crystallographic texture data set is discussed in the second subsection. By using this experimental EBSD technique, the different EBSD data sets representing the deformation states of a tensile specimen of the heat-treated DC04 steel are shown. In addition, a Matlab toolbox MTEX allowing to import the EBSD data formats and to obtain an image of the grain structure is introduced. In the third subsection, the microstructural image of the tensile specimen at the initial state is discretized by finite elements. The FE mesh is used as an input data for performing crystal plasticity simulations on the grain scale into the ABAQUS/CAE software.

\section{Constitutive model on a single crystal Elastic law}

An elastoviscoplastic constitutive model on a single crystal is introduced. The model is described in the context of large strain theory. Conceptually, the model is relied on the assumption of small elastic strains, finite plastic strains, and rotations. Plastic deformation is assumed to be the result from distinct slip mechanisms on specific crystallographic planes. The theory was developed in the works of $[2,4]$. The deformation gradient is decomposed multiplicatively into an elastic part $\boldsymbol{F}_{e}$ and a plastic part $\boldsymbol{F}_{p}[11,12]$

$$
\boldsymbol{F}=\boldsymbol{F}_{e} \boldsymbol{F}_{p}
$$

The plastic deformation $\boldsymbol{F}_{p}$ is the plastic contribution from crystallographic slips. The elastic deformation $\boldsymbol{F}_{e}$ accounts for the lattice distortion, which is inherently elastic. As the elastic strains are assumed to be small, a linearized relation between a conjugate pair 
of stress and strain measures is applicable for the description of the elastic behavior. Here, the elastic law is assumed to be given by

$$
\boldsymbol{\tau}=\boldsymbol{F}_{e} \tilde{\mathbb{C}}\left[\boldsymbol{E}_{e}\right] \boldsymbol{F}_{e}^{T} .
$$

The Kirchhoff stress tensor is given by $\boldsymbol{\tau}=\operatorname{det}(\boldsymbol{F}) \boldsymbol{\sigma}$, with $\boldsymbol{\sigma}$ being the Cauchy stress tensor. Green's strain tensor is defined by

$$
\boldsymbol{E}_{e}=\left(\boldsymbol{C}_{e}-\boldsymbol{I}\right) / 2,
$$

with $I$ being the second-order unit tensor and the right (elastic) Cauchy-Green tensor

$$
\boldsymbol{C}_{e}=\boldsymbol{F}_{e}^{\mathrm{T}} \boldsymbol{F}_{e} .
$$

The reference stiffness tensor $\tilde{\mathbb{C}}$ with respect to the orthonormal basis $\boldsymbol{B}_{\alpha}$ is given by

$$
\tilde{\mathbb{C}}=\tilde{C}_{\alpha \beta} \boldsymbol{B}_{\alpha} \otimes \boldsymbol{B}_{\beta}=\left[\begin{array}{cccccc}
C_{1111} & C_{1122} & C_{1122} & 0 & 0 & 0 \\
& C_{1111} & C_{1122} & 0 & 0 & 0 \\
& & C_{1111} & 0 & 0 & 0 \\
& & & 2 C_{1212} & 0 & 0 \\
& & & & 2 C_{1212} & 0 \\
& & & & & 2 C_{1212}
\end{array}\right] \boldsymbol{B}_{\alpha} \otimes \boldsymbol{B}_{\beta} .
$$

The components $\tilde{C}_{\alpha \beta}$ are defined by $\tilde{C}_{\alpha \beta}=\boldsymbol{B}_{\alpha} \cdot \tilde{\mathbb{C}}\left[\boldsymbol{B}_{\beta}\right]$. The orthonormal base tensors $\boldsymbol{B}_{\alpha}$ used are given by [13]. Due to the cubic material under consideration, the stiffness tensor $\widetilde{\mathbb{C}}$ has three independent elastic constants.

\section{Flow rule and hardening law}

A rate-dependent flow rule specifies the time evolution of the plastic part $\boldsymbol{F}_{p}$ of $\boldsymbol{F}$

$$
\dot{\boldsymbol{F}}_{p} \boldsymbol{F}_{p}^{-1}=\sum_{\alpha} \dot{\gamma}_{\alpha} \tilde{\boldsymbol{M}}_{\alpha}, \quad \dot{\gamma}_{\alpha}=\dot{\gamma}_{0} \operatorname{sgn}\left(\tau_{\alpha}\right)\left|\frac{\tau_{\alpha}}{\tau^{C}}\right|^{m},
$$

where the exponent $m$ quantifies the strain rate sensitivity of the material, $\dot{\gamma}_{0}$ is a reference rate, and $\tilde{\boldsymbol{M}}_{\alpha}$ is the Schmid tensor. $\tau^{C}$ denotes the critical resolved shear stress. A ratedependent Kocks-Mecking hardening model see, e.g., [7,14]

$$
\dot{\tau}^{C}\left(\tau_{\alpha}, \tau^{C}\right)=\Theta_{0}\left(1-\frac{\tau^{C}}{\tau_{V}^{C}\left(\tau_{\alpha}, \tau^{C}\right)}\right) \dot{\gamma}\left(\tau_{\alpha}, \tau^{C}\right)
$$

is used, where the critical Voce stress is specified by

$$
\tau_{V}^{C}\left(\tau_{\alpha}, \tau^{C}\right)=\tau_{V 0}^{C}\left(\frac{\dot{\gamma}\left(\tau_{\alpha}, \tau^{C}\right)}{\dot{\gamma}_{0}}\right)^{\frac{1}{n}}
$$

with the asymptotic critical resolved shear stress $\tau_{V 0}^{C}$ and the initial hardening modulus $\Theta_{0}$. The rate of the accumulated plastic slip is computed by

$$
\dot{\gamma}=\sum_{\alpha}\left|\dot{\gamma}_{\alpha}\left(\tau_{\alpha}, \tau^{C}\right)\right| .
$$

The resolved shear stress is defined by

$$
\tau_{\alpha}=\boldsymbol{T}_{e}^{\prime} \cdot \tilde{\boldsymbol{M}}_{\alpha},
$$

where

$$
T_{e}=C_{e} S_{e}^{2 \mathrm{PK}}
$$


denotes the Mandel stress tensor. The second Piola-Kirchhoff in the undistorted state is given by $\boldsymbol{S}_{e}^{2 \mathrm{PK}}=J \boldsymbol{F}_{e}^{-1} \boldsymbol{\tau} \boldsymbol{F}_{e}^{-\top} . J=\operatorname{det}\left(\boldsymbol{F}_{e}\right)$ is the determinant of $\boldsymbol{F}_{e}$. The Schmid or slip system tensors are rank-one tensors, which are defined in terms of the slip direction $\tilde{\boldsymbol{d}}_{\alpha}$ and slip plane normal $\tilde{\boldsymbol{n}}_{\alpha}$ in the undistorted configuration

$$
\tilde{\boldsymbol{M}}_{\alpha}=\tilde{\boldsymbol{d}}_{\alpha} \otimes \tilde{\boldsymbol{n}}_{\alpha} .
$$

The initial conditions for the ordinary differential equation are $\boldsymbol{F}_{e}(0)=\boldsymbol{Q}(t=0) \in S O(3)$ and the initial critical resolved shear stress $\tau^{C}(0)=\tau_{0}^{C}$. The crystal orientation is given by a proper orthogonal tensor $\boldsymbol{Q}(t)=\boldsymbol{g}_{i}(t) \otimes \boldsymbol{e}_{i}$, where the vectors $\boldsymbol{g}_{i}$ and $\boldsymbol{e}_{i}$ denote the orthonormal lattice vectors and the fixed orthonormal basis, respectively. The initial orientation of the single crystal $\boldsymbol{Q}(t=0)=\boldsymbol{g}_{i}(0) \otimes \boldsymbol{e}_{i}$ is defined in terms of the orthonormal lattice vectors $\boldsymbol{g}_{i}(0)$ at the time $t=0$. As shown in [15], intrinsic characteristics of bodycentered cubic $(\mathrm{BCC})$ crystals are revealed by using a proper parameter identification method. The authors applied a $\mathrm{BCC}$ crystal plasticity model to perform uniaxial tension simulations at the material point level for different types of BCC single crystals and compare these with experiments. The results indicate that $\{110\}$ and $\{112\}$ planes are identified as intrinsic slip systems of BCC crystals, but not the $\{123\}$ plane. Therefore, in this work, the attention is focused on a combination of $\{110\}\langle 111\rangle$ and $\{112\}\langle 111\rangle$ slip system families (Figure 1). There are two slip directions in each of the slip planes along the main diagonals of the cube. In total, there are 24 slip systems shown in Tables 1 and 2.

\section{Experimental identification based on EBSD data of DC04 steel tensile samples EBSD measurement}

In recent years, EBSD has become an important technique for the quantitative characterization of different microstructural properties such as the grain size, the grain boundary structure, and the orientation distribution (see, e.g., [17-19]). The main objective of the technique is to obtain spatially resolved crystallographic information by a SEM. For every point analyzed on a sample, the position, the phase, and the orientation information are stored. The stored data set is a database of measurements produced by scanning the beam in a regular grid over the sample. The data format is shown in Figure 2 (left) for the microstructure sample of the mentioned DC04 steel at the initial state. Each row is a measurement point in the map, and each column is one of the several measured parameters. In the first column, each match unit contains the information necessary to model the EBSD pattern produced by the expected phase in the sample. The phase values 1 indicate the expected phases of ferrite, while the phase values 0 correspond to measurement errors.

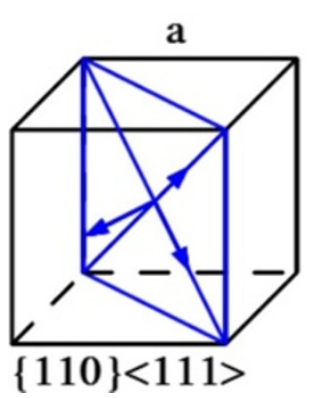

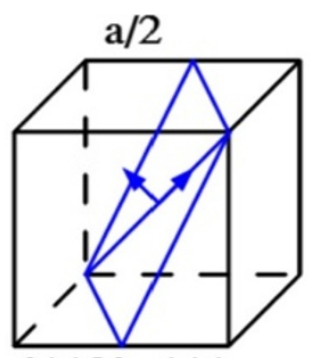

$\{112\}<111>$

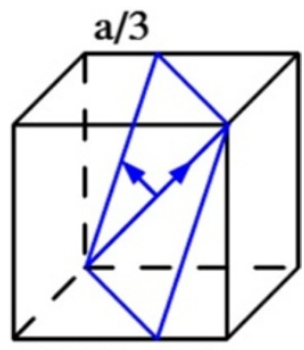

$\{123\}<111>$

Figure 1 BCC slip systems of the unit cell (cubic crystal). 
Table 112 BCC slip systems of $\{110\}\langle 111\rangle[16]$

\begin{tabular}{ccccccc}
\hline \multicolumn{7}{c}{ Slip system $(\boldsymbol{\alpha})$} \\
\hline$\sqrt{2}\left[\tilde{n}_{i}^{\alpha}\right]$ & 1 & 2 & 3 & 4 & 5 & 6 \\
$\sqrt{3}\left[\tilde{d}_{i}^{\alpha}\right]$ & {$[1,-1,0]$} & {$[1,-1,0]$} & {$[1,0,-1]$} & {$[1,0,-1]$} & {$[0,1,-1]$} & {$[0,1,-1]$} \\
& {$[1,1,1]$} & {$[1,1,-1]$} & {$[1,1,1]$} & {$[1,-1,1]$} & {$[1,1,1]$} & {$[-1,1,1]$} \\
$\sqrt{2}\left[\tilde{n}_{i}^{\alpha}\right]$ & 7 & 8 & 9 & 10 & 11 & 12 \\
$\sqrt{3}\left[\tilde{d}_{i}^{\alpha}\right]$ & {$[1,1,0]$} & {$[1,1,0]$} & {$[1,0,1]$} & {$[1,0,1]$} & {$[0,1,1]$} & {$[0,1,1]$} \\
\hline
\end{tabular}

The second and third column are the $X$ and $Y$ positions of the measurement points. Their dimensional unit is micrometer $[\mu \mathrm{m}]$. The rotation is commonly parameterized by a triple of Euler angles $\phi_{1}, \Phi, \phi_{2}$ and is described through a $3 \times 3$ orthogonal matrix given by

$$
\begin{aligned}
Q_{i j} & =\left[\begin{array}{ccc}
\cos \phi_{1} & -\sin \phi_{1} & 0 \\
\sin \phi_{1} & \cos \phi_{1} & 0 \\
0 & 0 & 1
\end{array}\right]\left[\begin{array}{ccc}
1 & 0 & 0 \\
0 & \cos \Phi & -\sin \Phi \\
0 & \sin \Phi & \cos \Phi
\end{array}\right]\left[\begin{array}{ccc}
\cos \phi_{2} & -\sin \phi_{2} & 0 \\
\sin \phi_{2} & \cos \phi_{2} & 0 \\
0 & 0 & 1
\end{array}\right] \\
& =\left[\begin{array}{ccc}
\cos \phi_{1} \cos \phi_{2}-\sin \phi_{1} \cos \Phi \sin \phi_{2} & -\cos \phi_{1} \sin \phi_{2}-\sin \phi_{1} \cos \Phi \cos \phi_{2} & \sin \Phi \sin \phi_{1} \\
\sin \phi_{1} \cos \phi_{2}+\cos \phi_{1} \cos \Phi \sin \phi_{2} & -\sin \phi_{1} \sin \phi_{2}+\cos \phi_{1} \cos \Phi \cos \phi_{2} & -\sin \Phi \cos \phi_{1} \\
\sin \Phi \sin \phi_{2} & \sin \Phi \cos \phi_{2} & \cos \Phi
\end{array}\right],
\end{aligned}
$$

where the three Euler angles $\phi_{1}, \Phi$, and $\phi_{2}$, shown schematically [20] in Figure 2 (right), are used to describe the crystallographic orientation of the crystals in relation to a reference coordinate system. In the database, these three Euler angles are recorded in the fourth, fifth, and sixth columns, respectively. The other parameters are neglected for the consideration.

\section{Identification of microstructures and orientation information}

An experimental tensile specimen of heat-treated low-carbon DC04 steel [21] is discussed here. The specimen geometry is width $w=5 \mathrm{~mm}$, gauge length $L_{0}=15 \mathrm{~mm}$, and thickness $t=1 \mathrm{~mm}$. From such specimen, a tiny sample was cut by laser rays with the same thickness. Through the EBSD technique in the scanning electron microscope, an initial raw database of such a sample is obtained.

Recently, a software package MTEX [22,23], a Matlab toolbox developed since 1997, is used for the quantitative analysis of experimental textures. The obtained EBSD database has been processed by MTEX to identify the grains and their boundaries. The EBSD sample database is then imported into the MTEX toolbox to identify the corresponding

Table 212 BCC slip systems of $\{112\}\langle 111\rangle$ [15]

\begin{tabular}{ccccccc}
\hline \multicolumn{7}{c}{ Slip system $(\boldsymbol{\alpha})$} \\
\hline$\sqrt{6}\left[\tilde{n}_{i}^{\alpha}\right]$ & 1 & 2 & 3 & 4 & 5 & 6 \\
$\sqrt{3}\left[\tilde{d}_{i}^{\alpha}\right]$ & {$[1,1,2]$} & {$[-1,1,2]$} & {$[1,-1,2]$} & {$[1,1,-2]$} & {$[1,2,1]$} & {$[-1,2,1]$} \\
& {$[1,1,-1]$} & {$[1,-1,1]$} & {$[-1,1,1]$} & {$[1,1,1]$} & {$[1,-1,1]$} & {$[1,1,-1]$} \\
$\sqrt{6}\left[\tilde{n}_{i}^{\alpha}\right]$ & 7 & 8 & 9 & 10 & 11 & 12 \\
$\sqrt{3}\left[\tilde{d}_{i}^{\alpha}\right]$ & {$[1,-2,1]$} & {$[1,2,-1]$} & {$[2,1,1]$} & {$[-2,1,1]$} & {$[2,-1,1]$} & {$[2,1,-1]$} \\
\hline
\end{tabular}




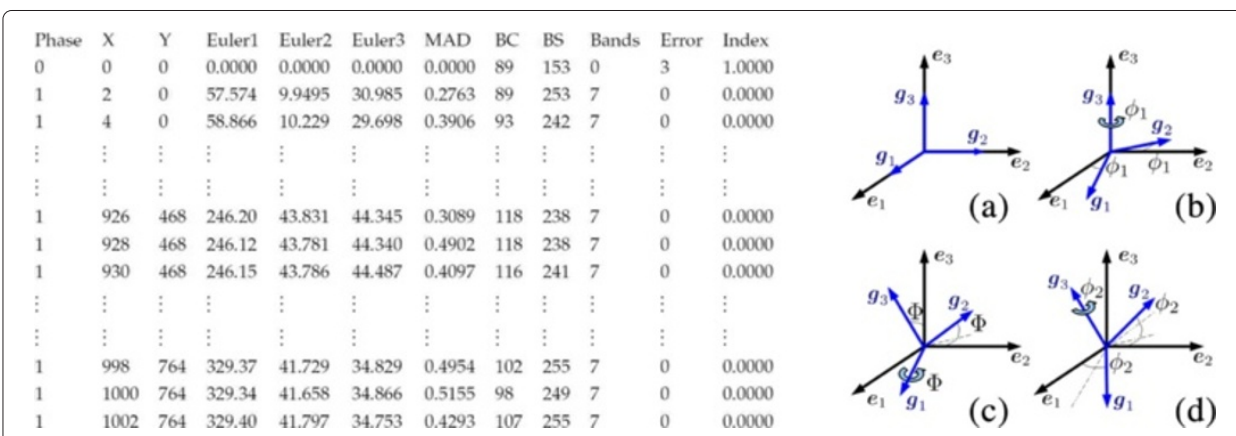

Figure $\mathbf{2}$ The file format and Euler angles. File format of the raw EBSD data set of the experimental sample at the initial state including 105,000 rows and 7 columns (left) and definition of Euler angles (right).

microstructure. Figure 3 shows an EBSD sample microstructure considered at the initial state. A strain rate of $10^{-3} \mathrm{~s}^{-1}$ is applied to the tensile specimen in loading direction. During the tensile test, several EBSD databases have been determined experimentally, and thereby, the evolution of the crystallographic texture is measured at different elongation states $(5 \%, 10 \%, 15 \%$, and $20 \%)$.

By using the MTEX toolbox, these raw EBSD databases are imported to obtain 2D images of grain structures. Two microstructural images of both the raw EBSD data and the clustered EBSD data are shown in Figure 4a,b, repectively, for the initial state of the sample. The grains consisting only of 1 pixel are eliminated in the clustering process of the MTEX toolbox. A clustered EBSD database which consists of identified grains and corresponding point sets is obtained. This database represents the $X$ and $Y$ positions (in $\mu \mathrm{m})$ and three Euler angles (in degree) of the measurement points. In addition, the grain

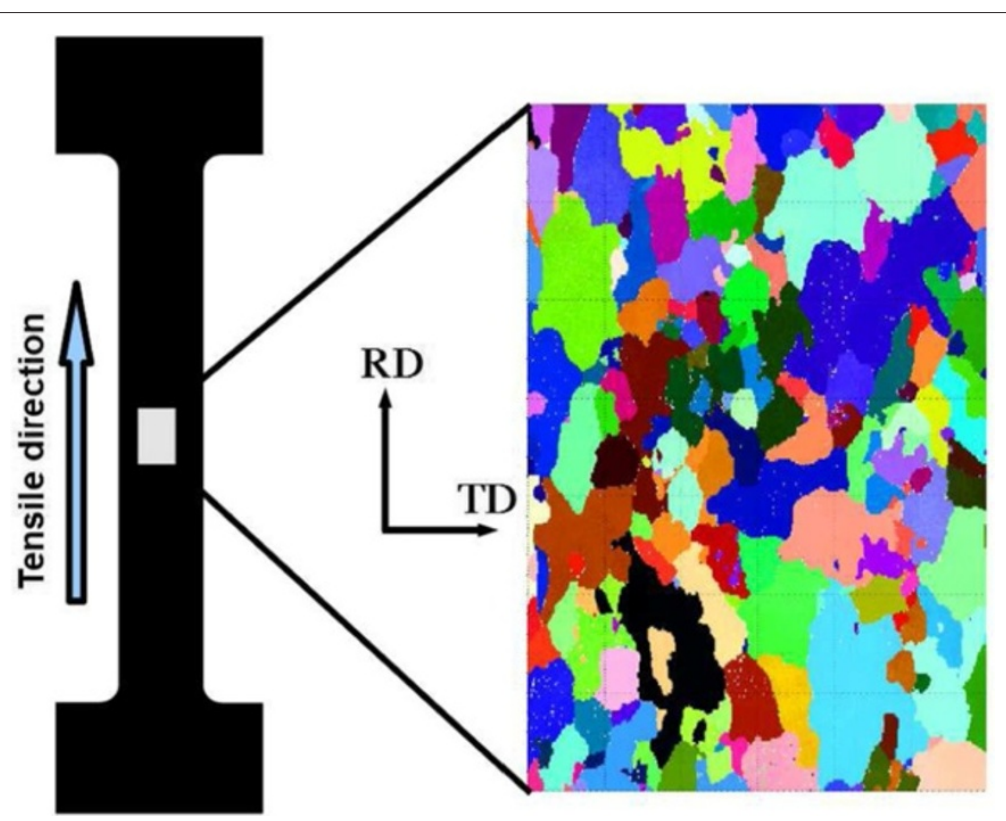

Figure $3 \mathrm{~A}$ raw microstructural image of the tiny DC04 steel sample. Raw EBSD microstructural image of a heterogeneous sample cut parallel to rolling direction from a DC04 steel specimen at the initial state. RD, rolling direction; TD, transverse direction. (For interpretation of the references to color in the text, the reader is referred to the web version of the article). 


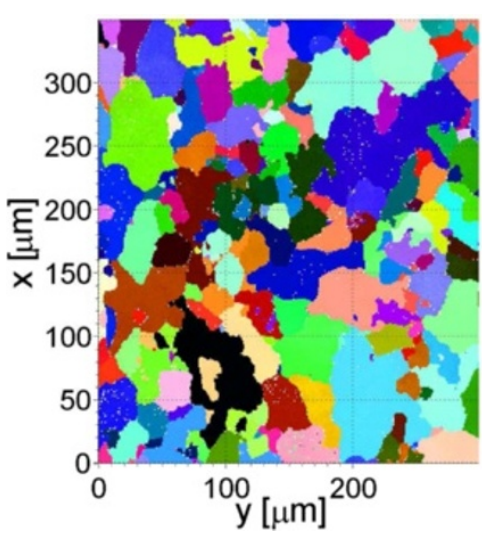

(a) Raw EBSD data set.

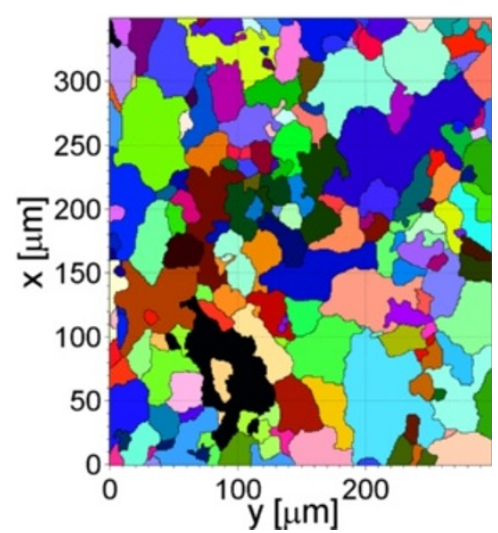

(b) Clustered EBSD data set.

Figure 4 Microstructural images at the initial state. (a) Raw EBSD data set. (b) Clustered EBSD data set.

to which the measurement points belong is indicated in the database. The order numbering of grains and the identification of 574 grains are shown in Figure 5 at the initial state. Additionally, 2D images of the raw EBSD data at different strain states $(5 \%, 10 \%, 15 \%$, and 20\%) are shown in Figure 6. In all microstructural images, each color indicates the lattice orientation in each grain at different states.

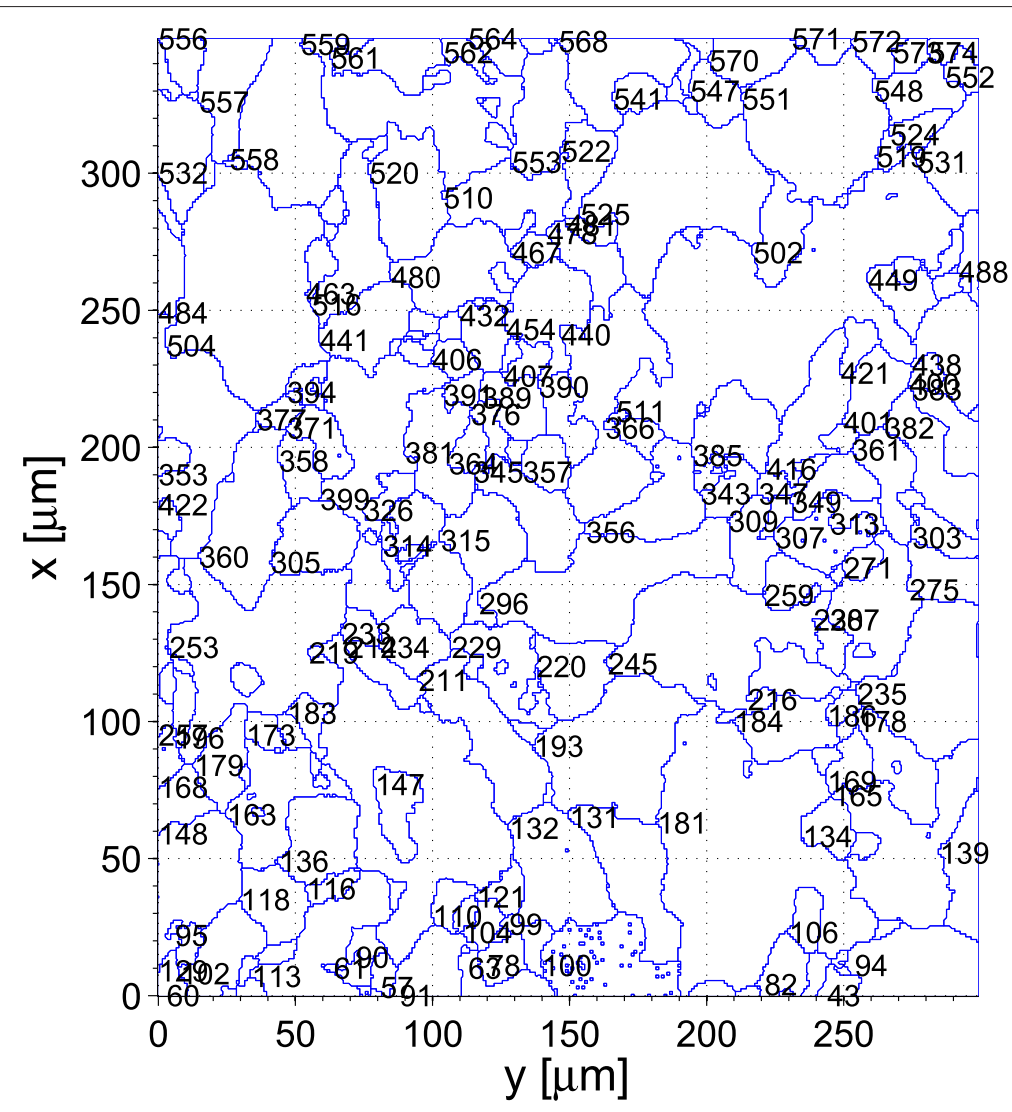

Figure 5 Identification and numbering of grains. Identification and numbering of 574 grains in the microstructural image of clustered data set at the initial state. 


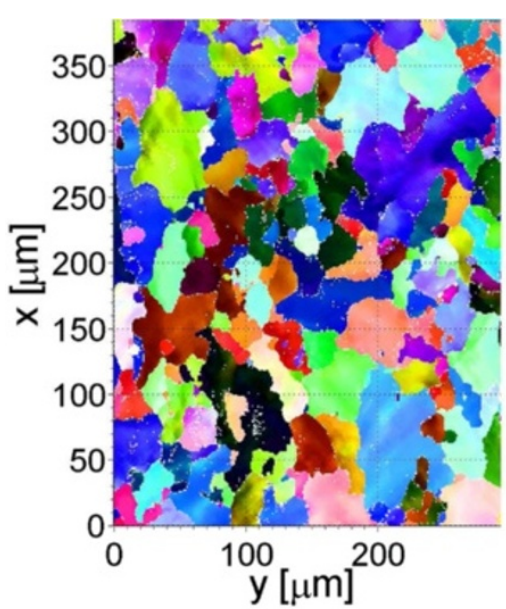

(a) $5 \%$ elongation.

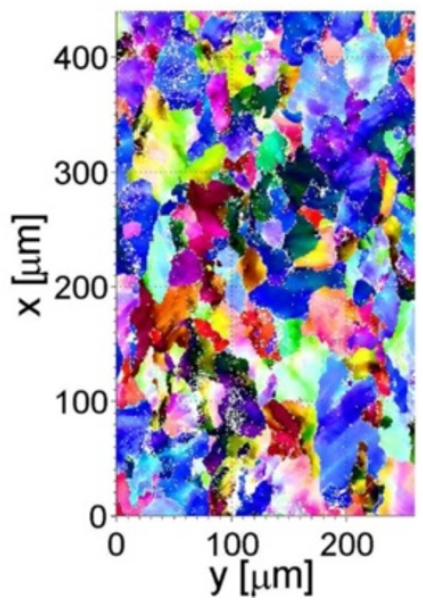

(c) $15 \%$ elongation.

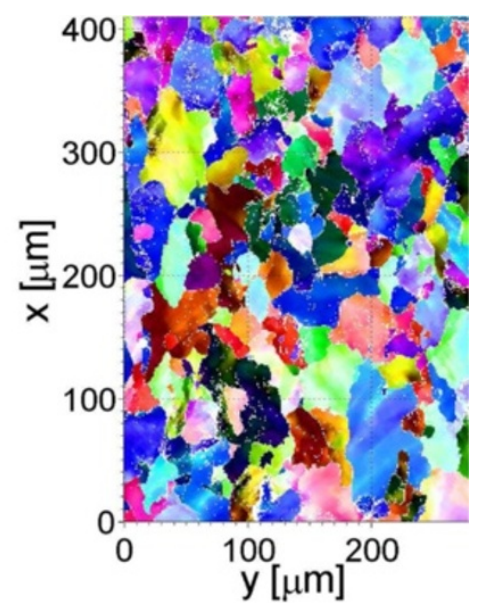

(b) $10 \%$ elongation.

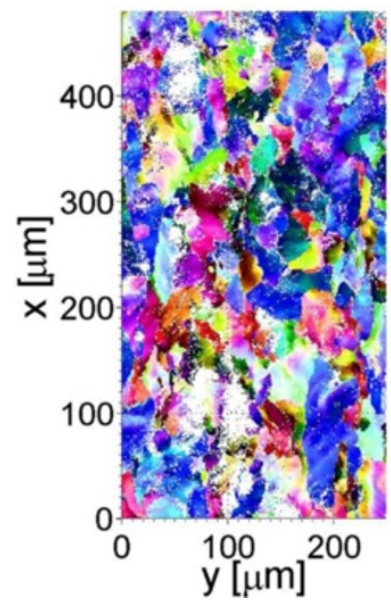

(d) $20 \%$ elongation.

Figure 6 Microstructural images of raw EBSD data sets at different states. (a) 5\% elongation. (b) 10\% elongation. (c) $15 \%$ elongation. (d) $20 \%$ elongation.

Some conclusive descriptions of the raw EBSD data are summarized. In the raw EBSD database of the initial state, the total number of columns and rows is 7 and 105,000, respectively. After neglecting measurement errors, in such an EBSD database remain 103,671 rows (or the number of pixels) and 7 columns. The number of measurement errors is 1,329 pixels, i.e., approximately $1.26 \%$ of the area fraction. The map size of the raw grain structure in Figure 4a is $349 \times 299 \mu \mathrm{m}$. The area of one measurement point is $1 \mu \mathrm{m}^{2}$. For the subsequent strain states, the measurement errors correspond to area fractions of $3.6 \%, 8.5 \%, 9.4 \%$, and $18.5 \%$, respectively.

\section{FE modeling and full-field simulation}

In this subsection, the microstructural image of the tensile specimen at the initial state shown in Figure 7 (left) is used as input data for performing crystal plasticity simulations on the grain scale. The sample picture is imported into a commercial software Simpleware to construct a computable grain structure model as shown in Figure 7 (right). Simpleware offers two important options for processing and meshing $2 \mathrm{D}$ or $3 \mathrm{D}$ image data. The 

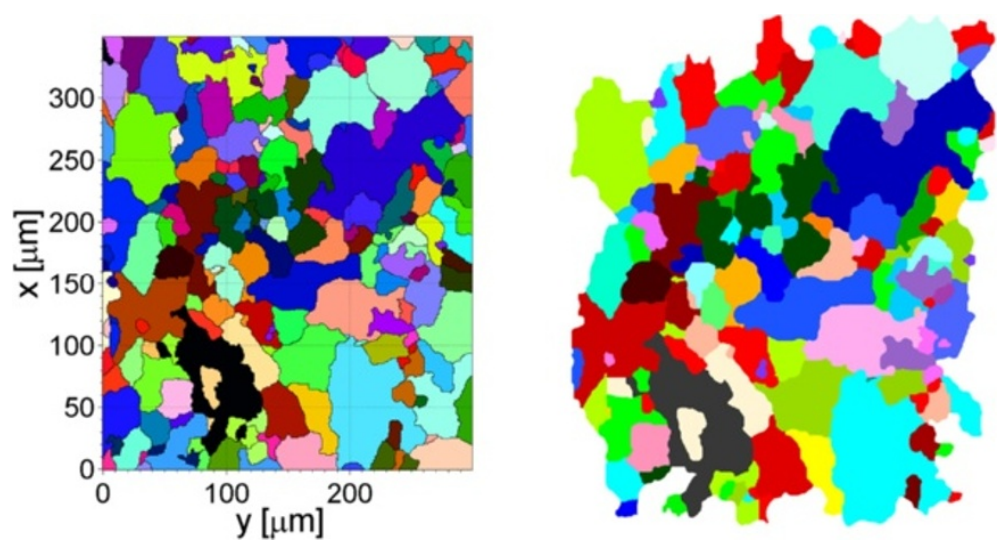

Figure 7 Microstructural image of the tensile specimen at the initial state. Microstructure of heat-treated DCO4 steel at the initial state obtained from the clustering process by MTEX toolbox (left) and a microstructure including complete grains constructed by Simpleware software (right).

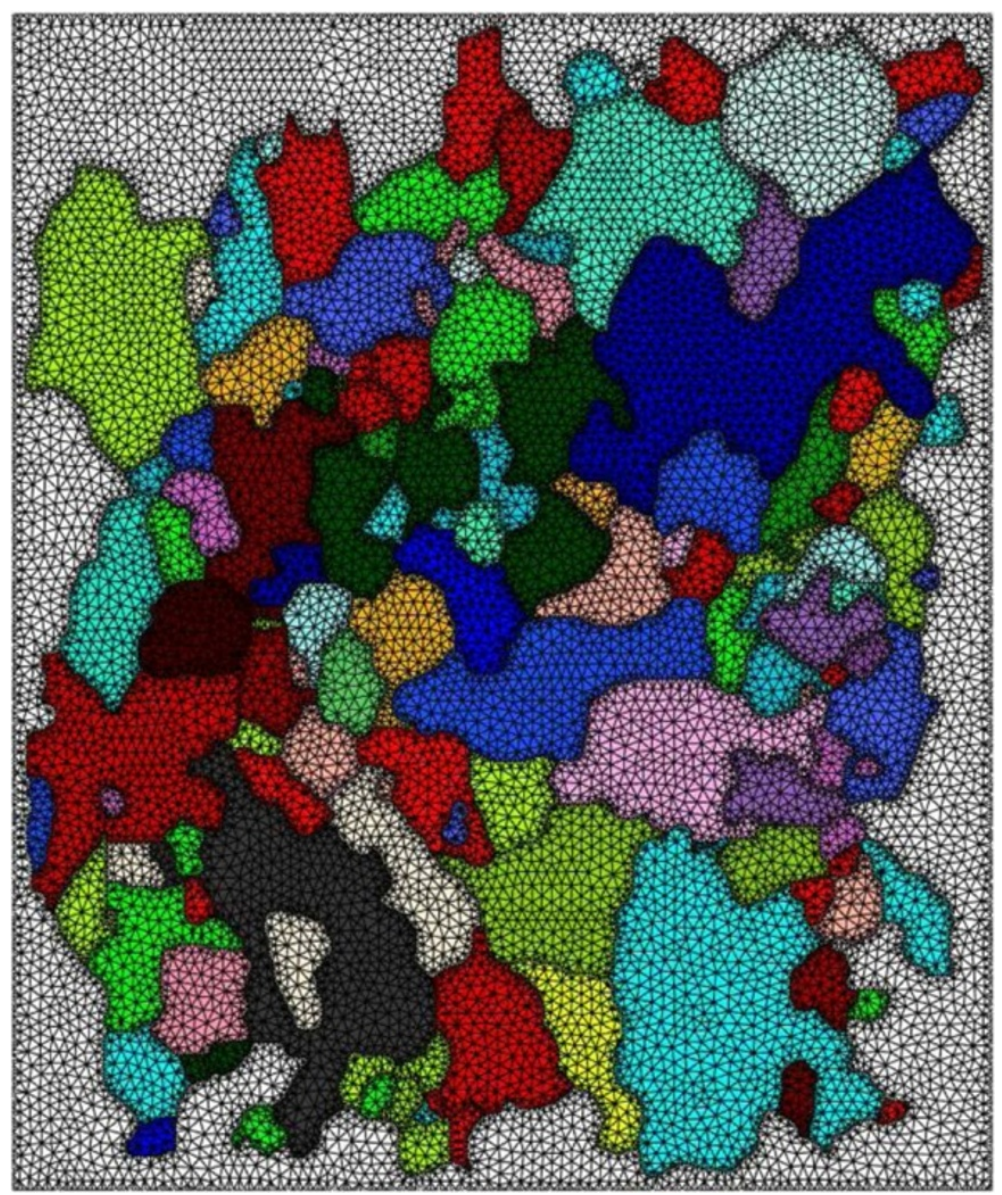

Figure $8 \mathrm{FE}$ model of the microstructure at the initial state. 


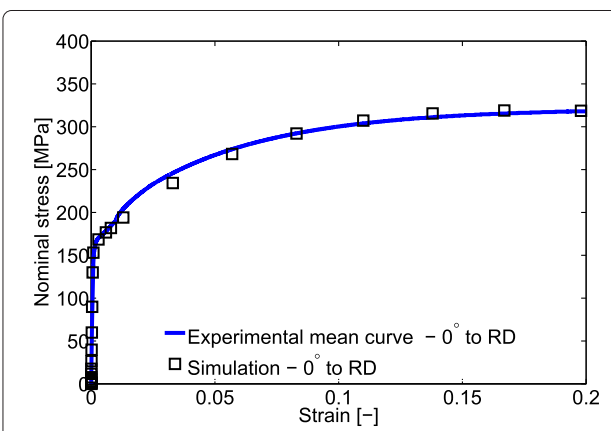

(a) $0^{\circ}$ to $\mathrm{RD}$

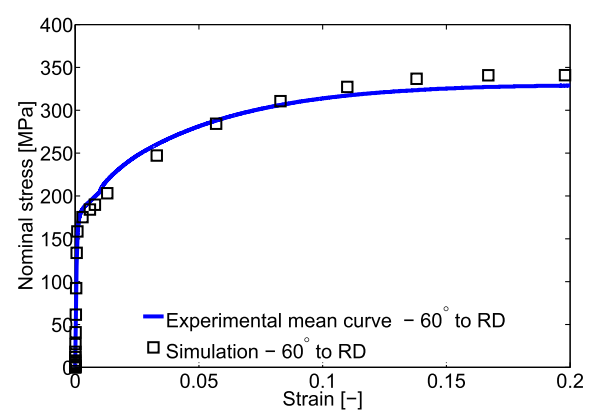

(c) $60^{\circ}$ to $\mathrm{RD}$

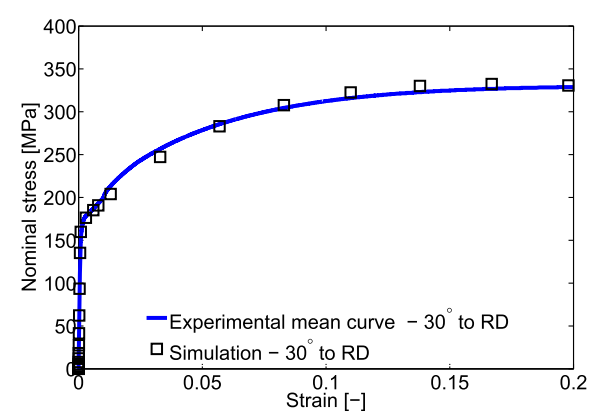

(b) $30^{\circ}$ to $\mathrm{RD}$

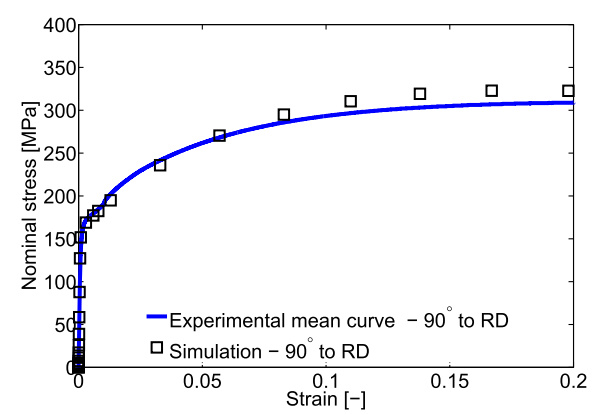

(d) $90^{\circ}$ to $\mathrm{RD}$

Figure 9 Stress-strain curves in comparison to experimental curves. Numerically determined stress-strain curves in comparison to experimental curves of the Institute of Forming Technology and Lightweight IUL (TU Dortmund University) for different angles to RD [25]. (a) $0^{\circ}$ to RD. (b) $30^{\circ}$ to RD. (c) $60^{\circ}$ to RD. (d) $90^{\circ}$ to RD.

first one is ScanIP, which is the platform for the image processing, and the second one is ScanFE, which is a fully integrated meshing module for the conversion of masks (or grains) to 2D or 3D FE meshes. The type of elements used in the FE mesh are linear and quadratic hexahedral elements. Details of the software can be found in [24]. Note that the color distribution of grains in Figure 7 (left) is not equal to the one in Figure 7 (right) due to the different conventions of colors between the MTEX and Simpleware software. After the processing steps in ScanIP, a FE output data containing the set of nodes, the set of hexahedral elements, and the set of tetrahedral elements is obtained. The data can be exported in the ABAQUS format with the FE mesh shown in Figure 8. Different colors indicate different crystal orientations of the grains. The white region around the grains
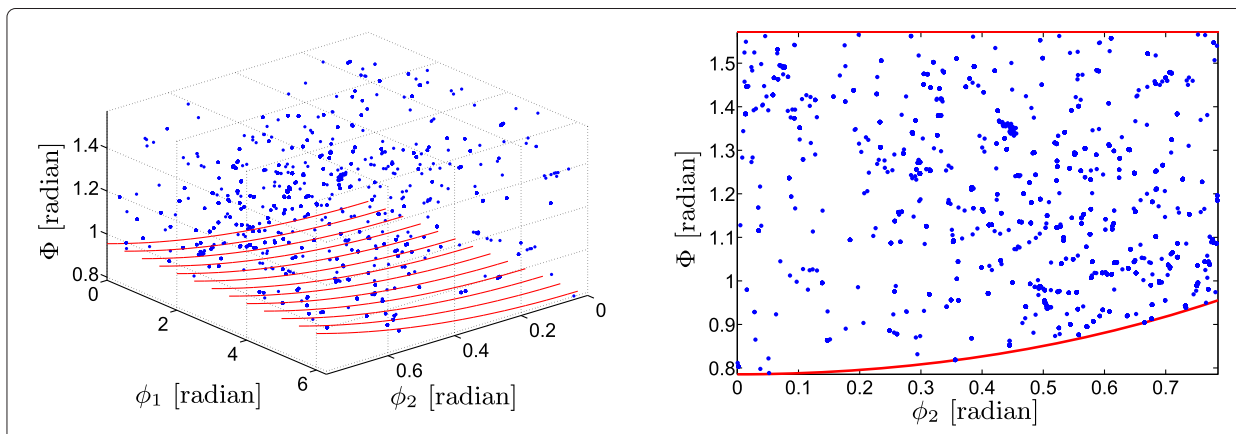

Figure 10 Initial grain orientations in the 3D (left) and 2D (right) fundamental zone $\phi_{2}-\Phi$. 


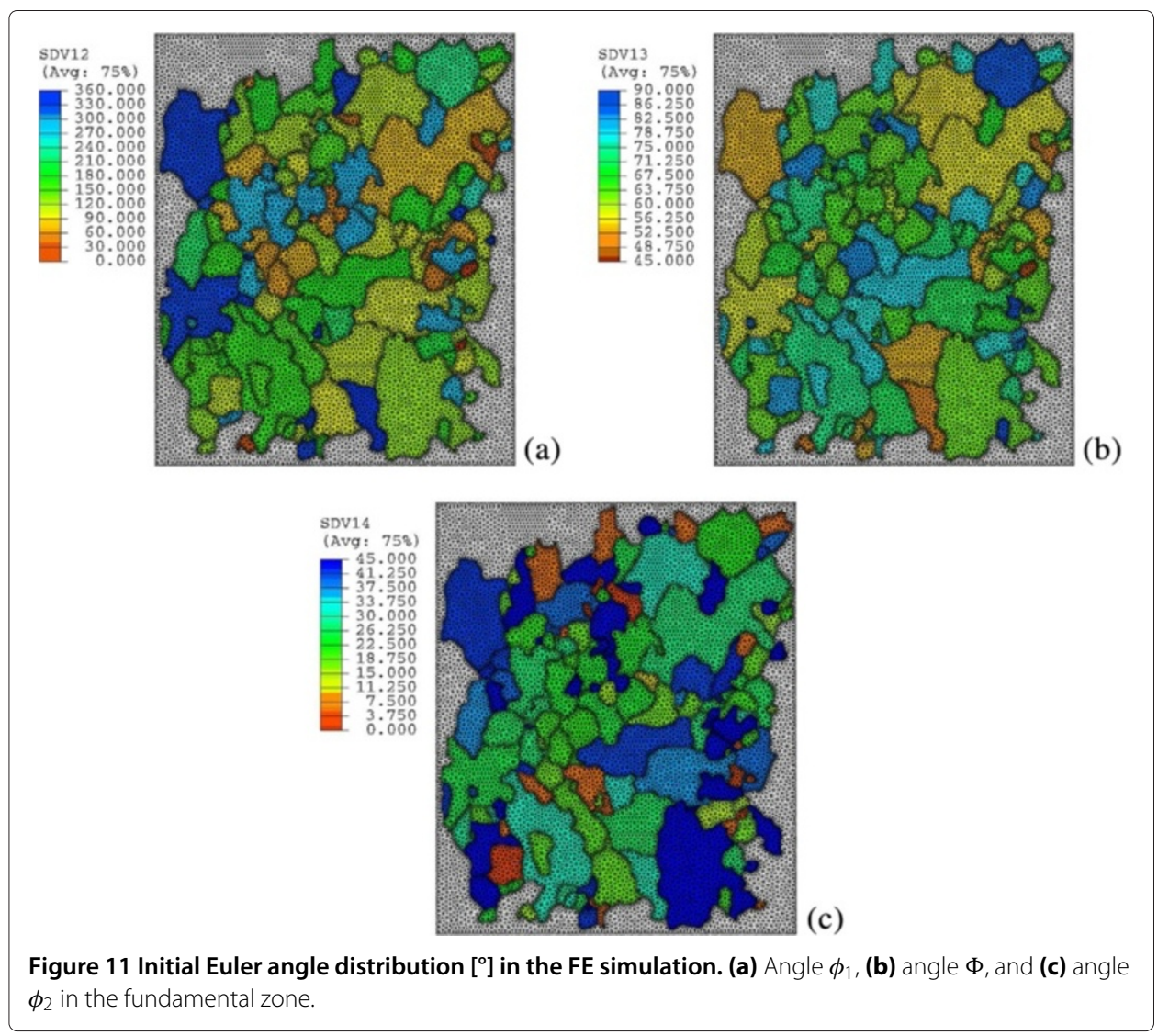

was assumed to show an ideal behavior according to the von Mises plasticity model. The elastic properties of steel with Young's modulus $(E=200 \mathrm{GPa})$ and Poisson's ratio $(v=0.3)$ are assigned to the ideal von Mises plastic region. For the plastic behavior, the flow parameters $\sigma_{F 0}=180 \mathrm{MPa}, \sigma_{F \infty}=303 \mathrm{MPa}$, describing the linear hardening, are estimated from the experimental tensile stress-strain curve for $0^{\circ}$ to the RD (Figure 9a).

In order to uniquely define the evolution of grains during the FE simulation, initial Euler angles of 45 chosen complete grains are transformed into the cubic fundamental zone. The triple of Euler angles restricted in the cubic fundamental zone is given by
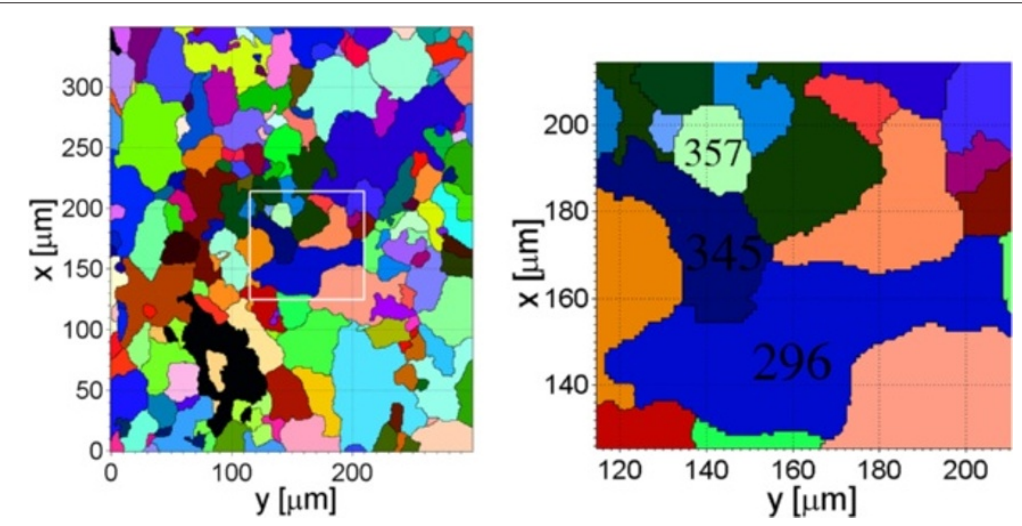

Figure 12 Location and enlargement of three local grains. Location of three selected local grains in the microstructural image (left). Enlargement of three local grains: \#296, \#345, and \#357 (right). 

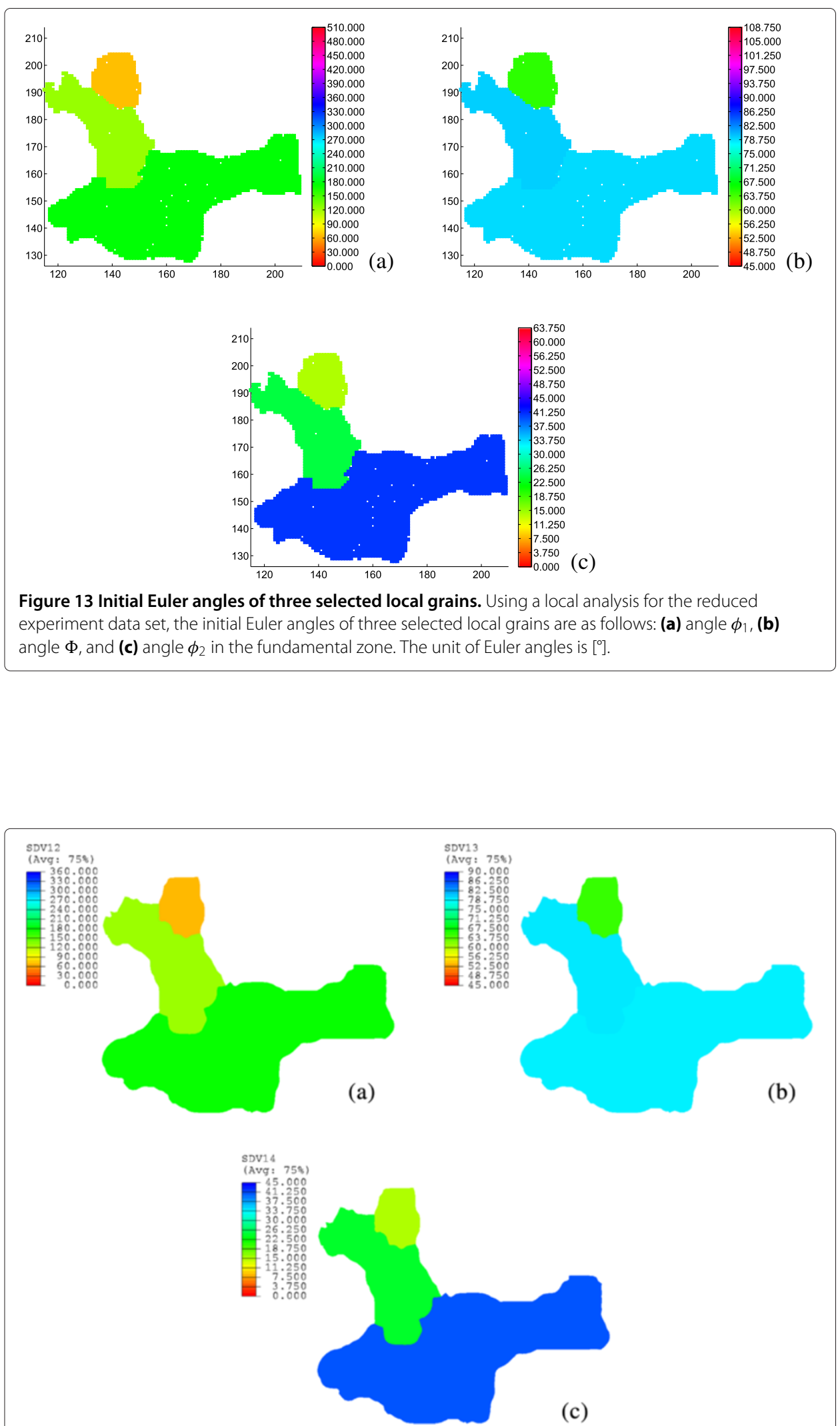

Figure 14 Initial Euler angles $\left[^{\circ}\right]$ of three local grains in the FE simulation. (a) Angle $\phi_{1}$, (b) angle $\Phi$, and (c) angle $\phi_{2}$ in the fundamental zone. 
Table 3 Set of material parameters

\begin{tabular}{lc}
\hline & Value \\
\hline Elastic constants of DC04 steel [27] & \\
$C_{1111}[\mathrm{GPa}]$ & 231.5 \\
$C_{1122}[\mathrm{GPa}]$ & 135.0 \\
$C_{1212}[\mathrm{GPa}]$ & 116.0 \\
Material parameters for flow and hardening rule [25] & \\
$\tau_{0}^{\mathrm{C}}[\mathrm{MPa}]$ & $67 \pm 6$ \\
$\tau_{V 0}^{C}[\mathrm{MPa}]$ & $130 \pm 3$ \\
$\Theta_{0}[\mathrm{MPa}]$ & $755 \pm 105$ \\
$m$ & 20 \\
$\dot{\gamma}_{0}\left[\mathrm{~s}^{-1}\right]$ & 0.001 \\
$n$ & 5 \\
\hline
\end{tabular}

$$
\begin{aligned}
\phi_{1} & \in[0,2 \pi) \\
\Phi & \in\left[f\left(\phi_{2}\right), \pi / 2\right] \\
\phi_{2} & \in[0, \pi / 4) \\
f\left(\phi_{2}\right) & =\arccos \left(\frac{\cos \left(\phi_{2}\right)}{\sqrt{1+\cos \left(\phi_{2}\right)^{2}}}\right) .
\end{aligned}
$$

The detailed explanation of the fundamental zone could be found in $[10,26]$. In Figure 10, the initial crystal orientations of the grains transformed in the fundamental zone are shown. In FE simulation, initial Euler angles of the aforementioned 45 grains are depicted in Figure 11. For the comparison about the grain orientation and reorientation between simulated results and experimental results, three local grains are chosen. Figure 12 shows the location of three local grains and their enlargement in the experimental microstructure. The experimental identification of three initial Euler angles of these three local grains is shown in Figure 13 after transformed in the fundamental zone. It can be seen that the initial Euler angles of these local grains are explicitly shown in the FE simulation (Figure 14) when compared to the identified experimental ones in the same scale color (RGB). The simulation is carried out by using the implicit Euler scheme for time integration and the user subroutine UMAT defining the constitutive law of the crystals introduced in the first subsection. The material parameters shown in Table 3 are used for

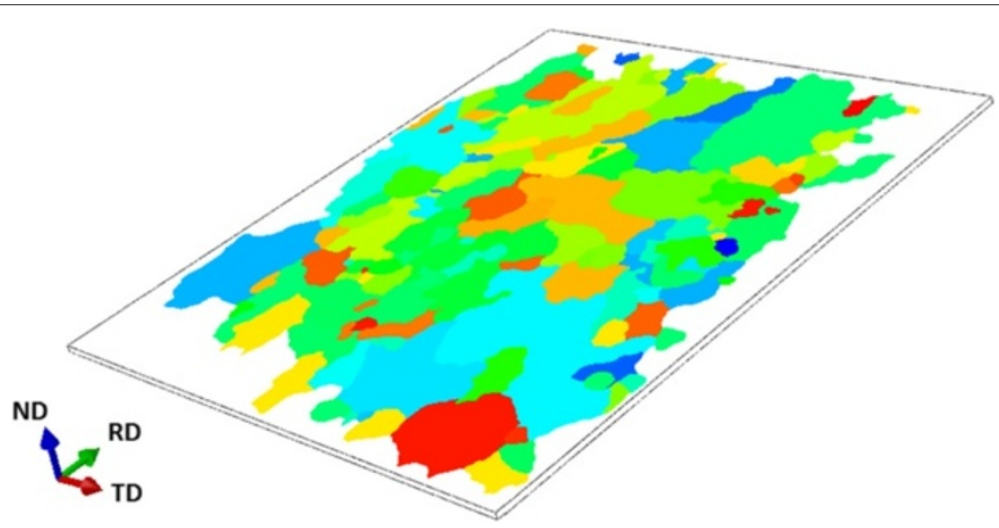

Figure 15 Reference coordinate system of microstructural sample. ND, normal direction; RD, rolling direction; TD, transverse direction. 


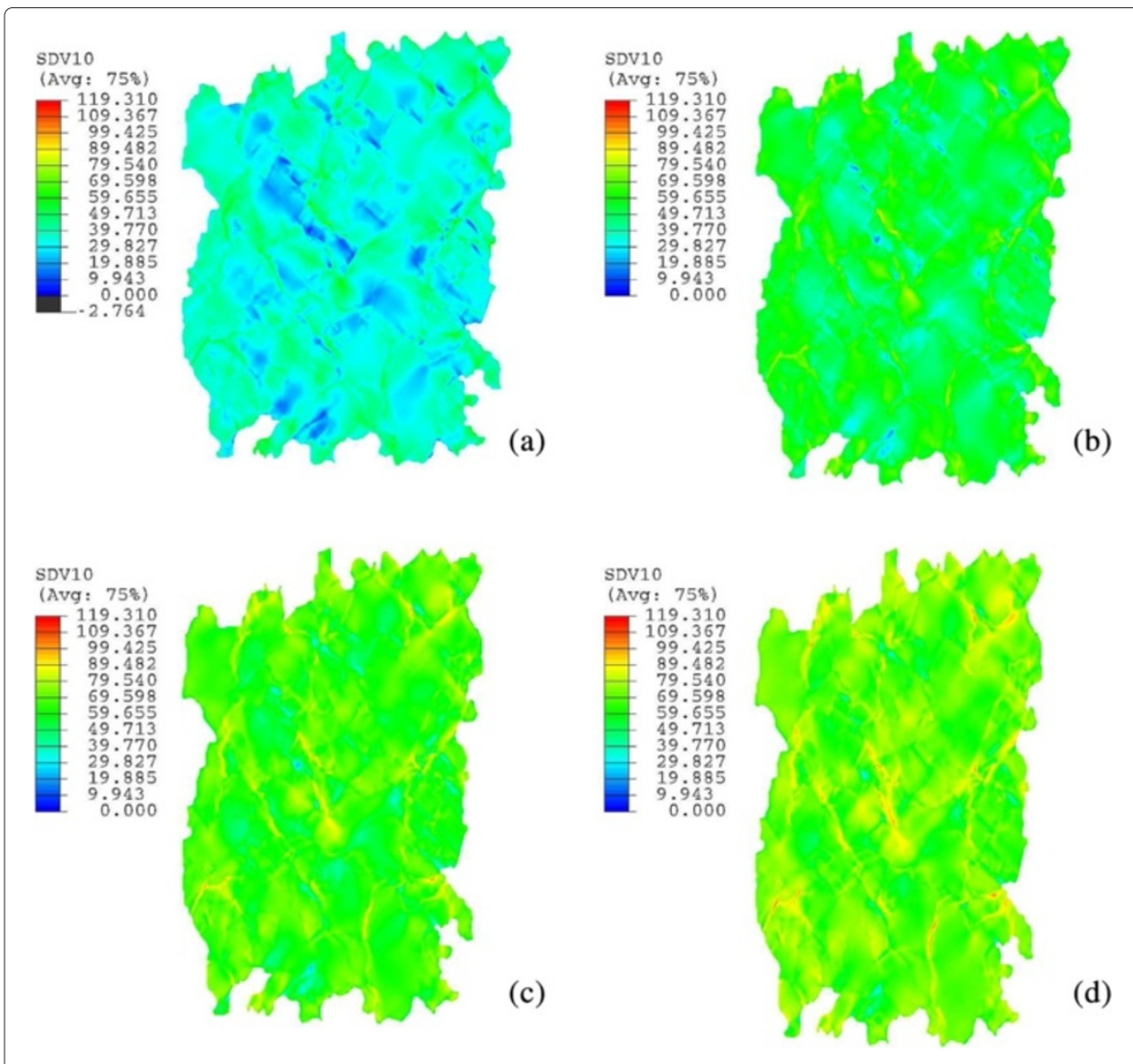

Figure 16 Distribution of critical resolved shear stress [MPa] of the grain structure at different states. (a) $5 \%$, (b) $10 \%$, (c) $15 \%$, and (d) $20 \%$ elongation.

performing the grain scale simulation. The set of these material parameters are identified by [25] by using tensile stress-strain curves at different angles to RD. The stress-strain curves are shown in Figure 9. For the slip mechanism, the grain scale simulation accounts for the combination of $\{110\}\langle 111\rangle+\{112\}\langle 111\rangle$ slip systems.

Considering in the reference coordinate system of microstructural sample (Figure 15), homogeneous displacement boundary conditions at the outer boundary of the RD-TD plane are applied. The strain in the normal direction (ND) is assumed to be zero. Furthermore, a plane strain state is assumed. The boundary conditions are defined by the ABAQUS subroutine DISP. The displacement vector and the displacement gradient are given by

$$
\boldsymbol{u}(X, t)=\boldsymbol{x}-\boldsymbol{X}
$$

and

$$
\boldsymbol{H}=\operatorname{Grad}(\boldsymbol{u}(\boldsymbol{X}, t)),
$$

respectively, where $\boldsymbol{X}$ is the reference position of the material points and $\boldsymbol{x}=\boldsymbol{\chi}_{\kappa}(\boldsymbol{X}, t)$ is the current position at time $t$. The displacement gradient $\boldsymbol{H}$ can be given in terms of the deformation gradient $\boldsymbol{F}$

$$
\boldsymbol{H}=\operatorname{Grad}(\boldsymbol{u}(\boldsymbol{X}, t))=\operatorname{Grad}(\boldsymbol{x})-\boldsymbol{I}=\boldsymbol{F}-\boldsymbol{I}
$$




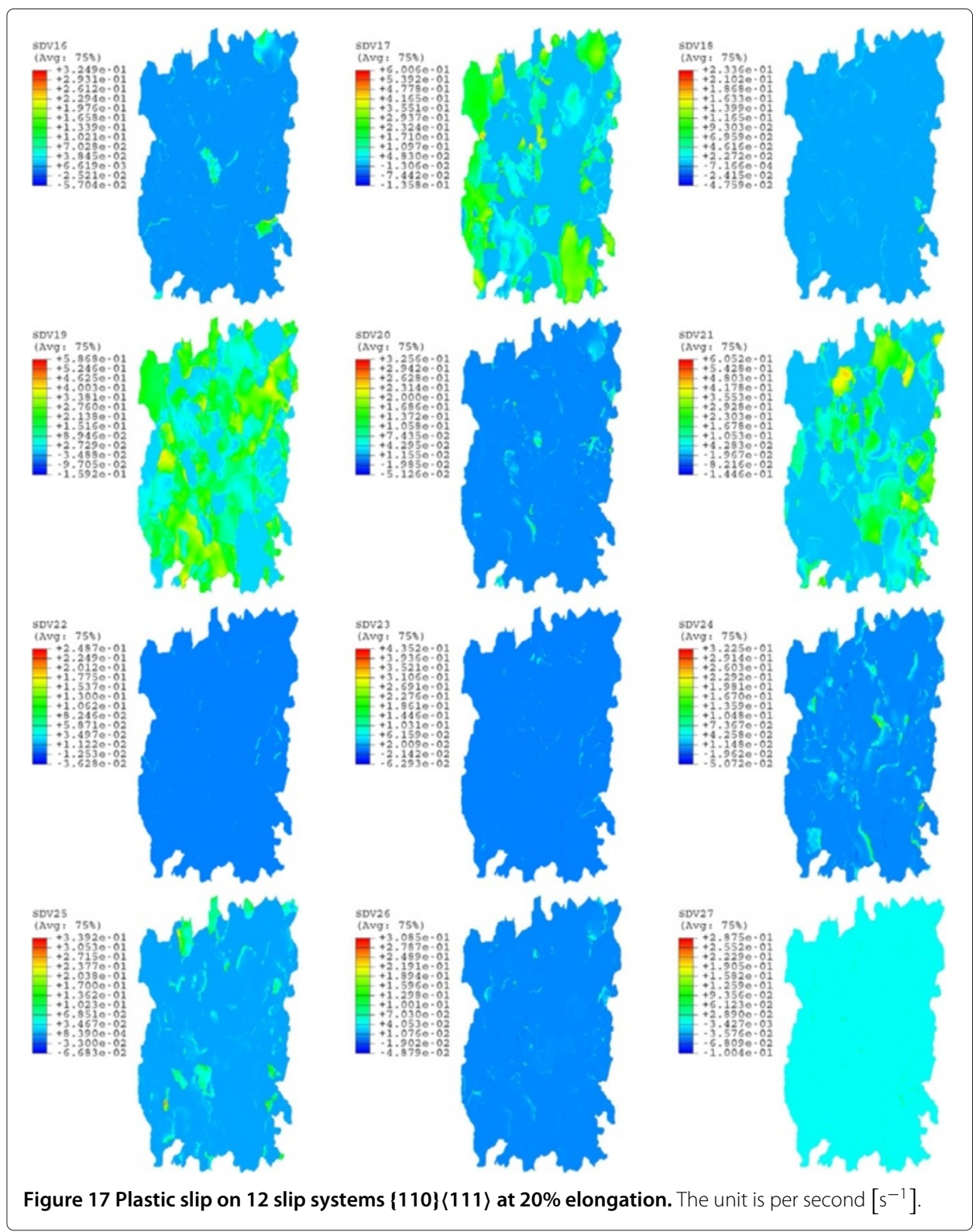

assuming the constant velocity gradient for plane strain compression is given by

$$
\boldsymbol{L}=\dot{\varepsilon}_{0}\left[\begin{array}{ccc}
\frac{-1}{\sqrt{2}} & 0 & 0 \\
0 & \frac{1}{\sqrt{2}} & 0 \\
0 & 0 & 0
\end{array}\right] \boldsymbol{e}_{i} \otimes \boldsymbol{e}_{j}
$$

The deformation gradient is given by the exponential form

$$
\boldsymbol{F}(t)=\exp (\boldsymbol{L} t) \boldsymbol{F}(0)
$$

with $\boldsymbol{F}(0)=\boldsymbol{I}$. The constant strain rate is set to $\dot{\varepsilon}_{0}=10^{-3} \mathrm{~s}^{-1}$. The special form of the velocity gradient implies a displacement in the $X-Y$ plane in the reference configuration 


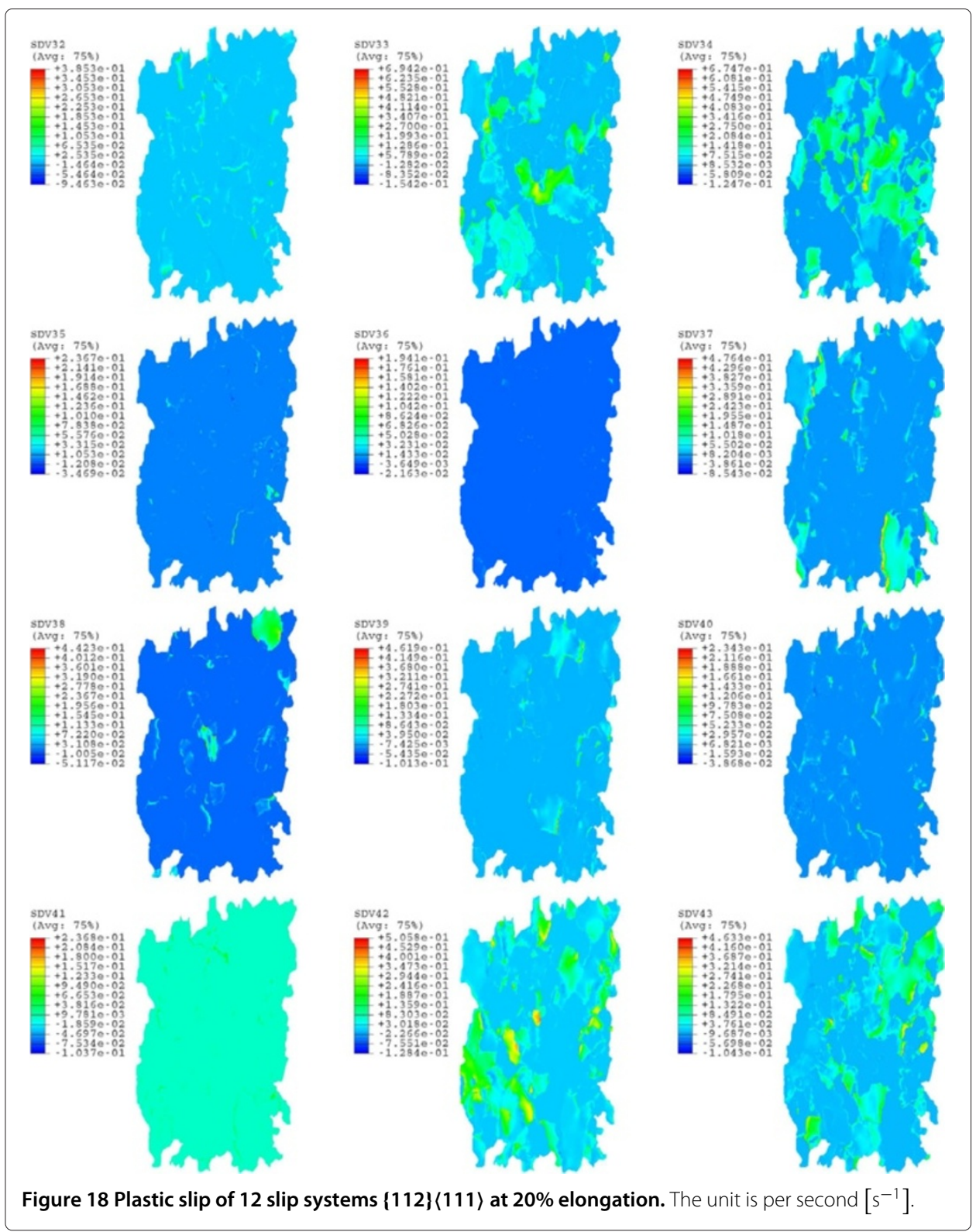

and a constant volume during the simulation. From Equation 20, the displacement on the boundary is obtained

$$
\boldsymbol{u}(\boldsymbol{X}, t)=\boldsymbol{H} \boldsymbol{X}=\boldsymbol{H} \boldsymbol{F}^{-1} \boldsymbol{x} .
$$

By combining this equation with Equation 21, the prescribed displacement is defined in the subroutine DISP at the time $t$ in terms of the deformation gradient in Equation 23, so that the displacement becomes

$$
\boldsymbol{u}(\boldsymbol{X}, t)=\left(\boldsymbol{I}-\boldsymbol{F}^{-1}\right) \boldsymbol{x} .
$$

The total time in FE simulation is $260 \mathrm{~s}$ corresponding to $20 \%$ elongation. The FE results are evaluated at different total times such as 65,130 , and $195 \mathrm{~s}$ corresponding to $5 \%, 10 \%$, and $15 \%$ elongation, respectively. The field of critical resolved shear stress $\tau_{c}$ is depicted in 

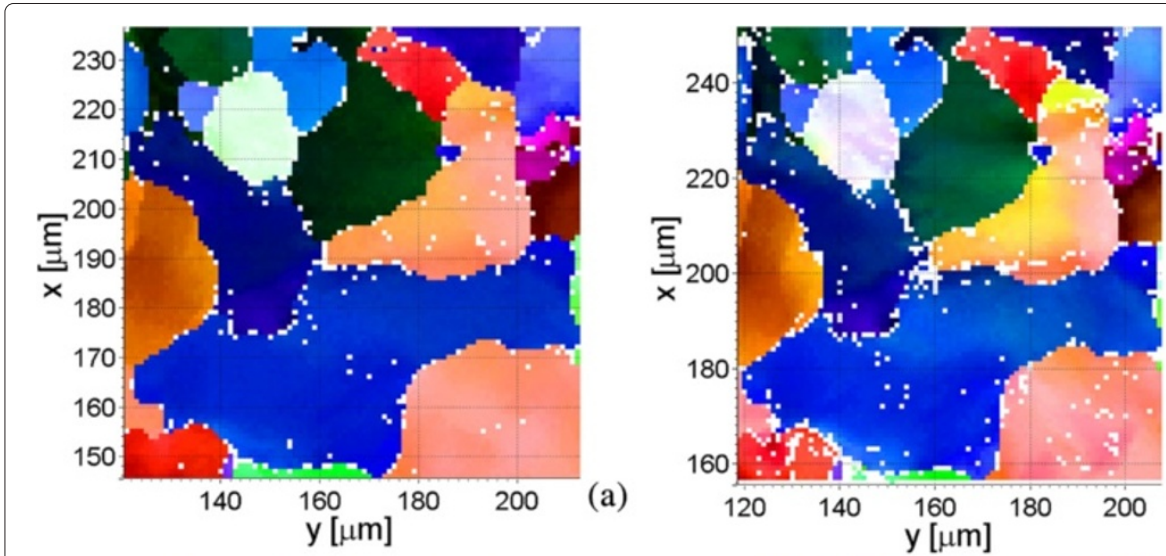

(b)
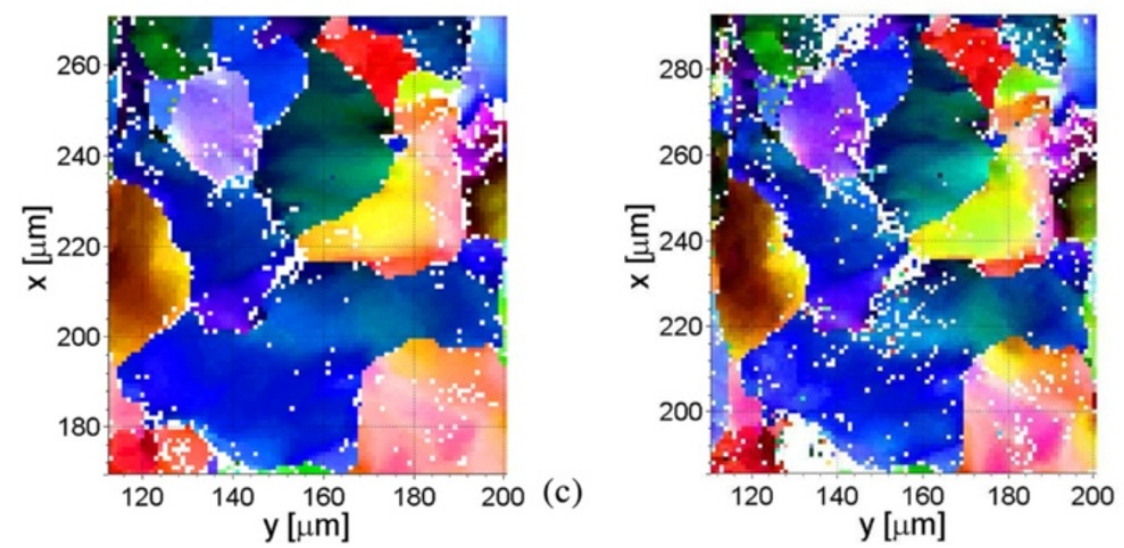

(d)

Figure 19 Microstructural images of local grains identified by MTEX at different states. (a) $5 \%$, (b) $10 \%$, (c) $15 \%$, and (d) $20 \%$ elongation.

Figure 16 at the aforementioned different states of the FE simulation. These results show a strongly inhomogeneous field caused by the evolution of individual grain orientations in the microstructure during deformation.

In addition, the plastic slip of each slip system $\alpha(\alpha=1 \ldots 24)$ in BCC slip systems $\{110\}\langle 111\rangle+\{112\}\langle 111\rangle$ is computed by integrating the slip rate $\dot{\gamma}_{\alpha}$ over the time during the grain scale simulation. The plastic slip is described as follows:

$$
\gamma_{\alpha}=\int_{0}^{t}\left|\dot{\gamma}_{\alpha}\right| \mathrm{d} t
$$

Figures 17 and 18 represent the plastic slip in 24 slip systems at 20\% elongation. It can be seen that the grain structure shows a very heterogeneous state of slip.

\section{Results and discussion}

This section aims to perform a comparison between the numerical results and the experimental data for three local grains. Numerical results at different tensile strains are compared to the corresponding experimental results. In order to obtain Euler angles and the reorientation in the three grains (Figure 19), post-processing steps have to be carried out. Firstly, the measured grain data set at each different state of deformation is transformed into the fundamental zone. Secondly, each triple of Euler angles of the measurement points is extracted at different states of deformation. A comparison of 


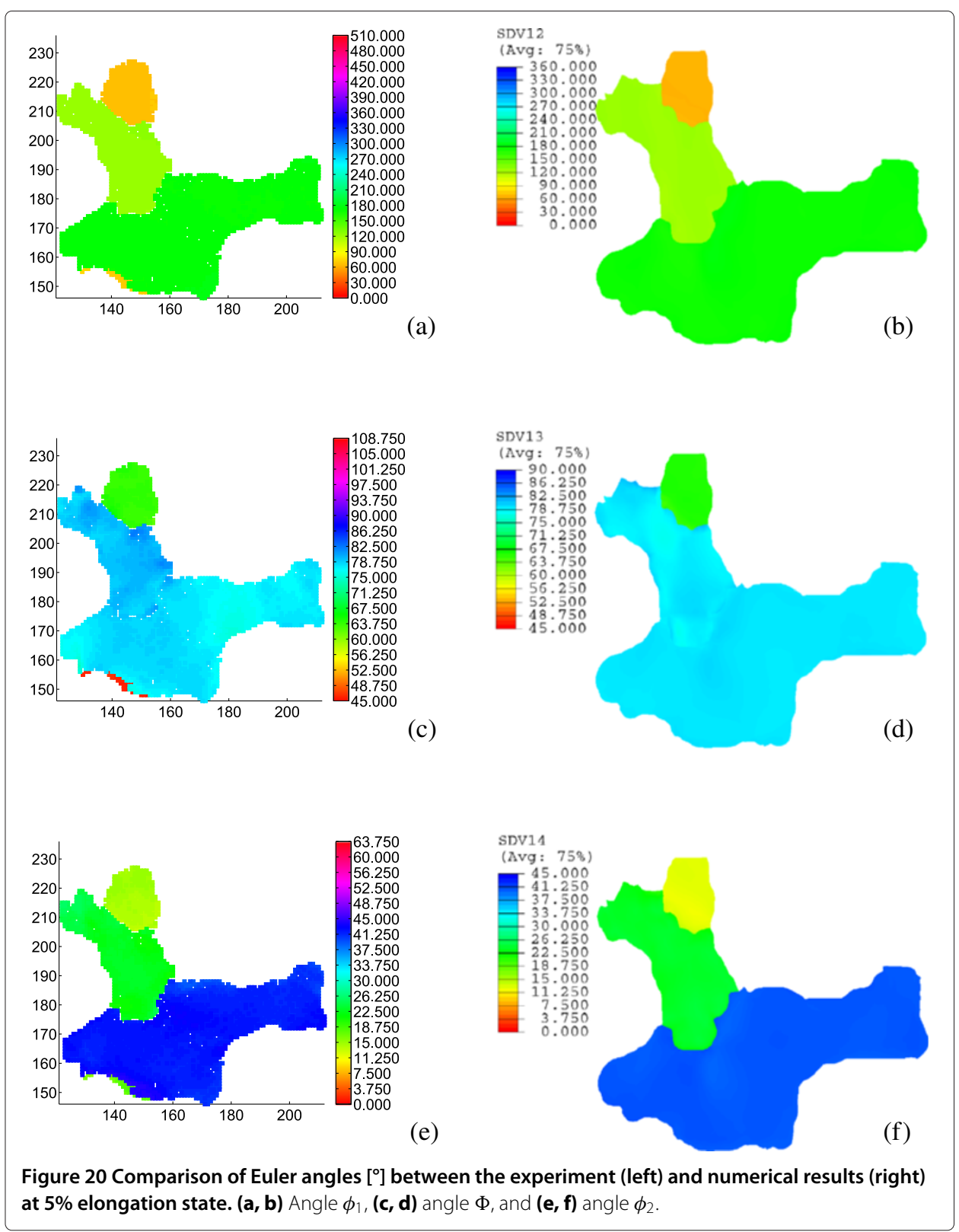

Euler angles between the experiment and the FE simulation is shown in Figures 20,21,22 and 23. The evolution of Euler angles in the numerical simulation is quite close to the experimental findings.

Thirdly, the reorientation of each measurement point is computed for each state of deformation. The formula to compute the angle of reorientation is given by

$$
\omega=\left|\arccos \left(\frac{\operatorname{tr} Q Q_{0}^{\top}-1}{2}\right)\right|,
$$

where $Q_{0}$ represents the crystal orientation at the initial state of deformation and $Q$ represents the crystal orientation of the same point at different states of deformation. Both $Q_{0}$ and $Q$ are parameterized by Euler angles lying in the same fundamental zone. 


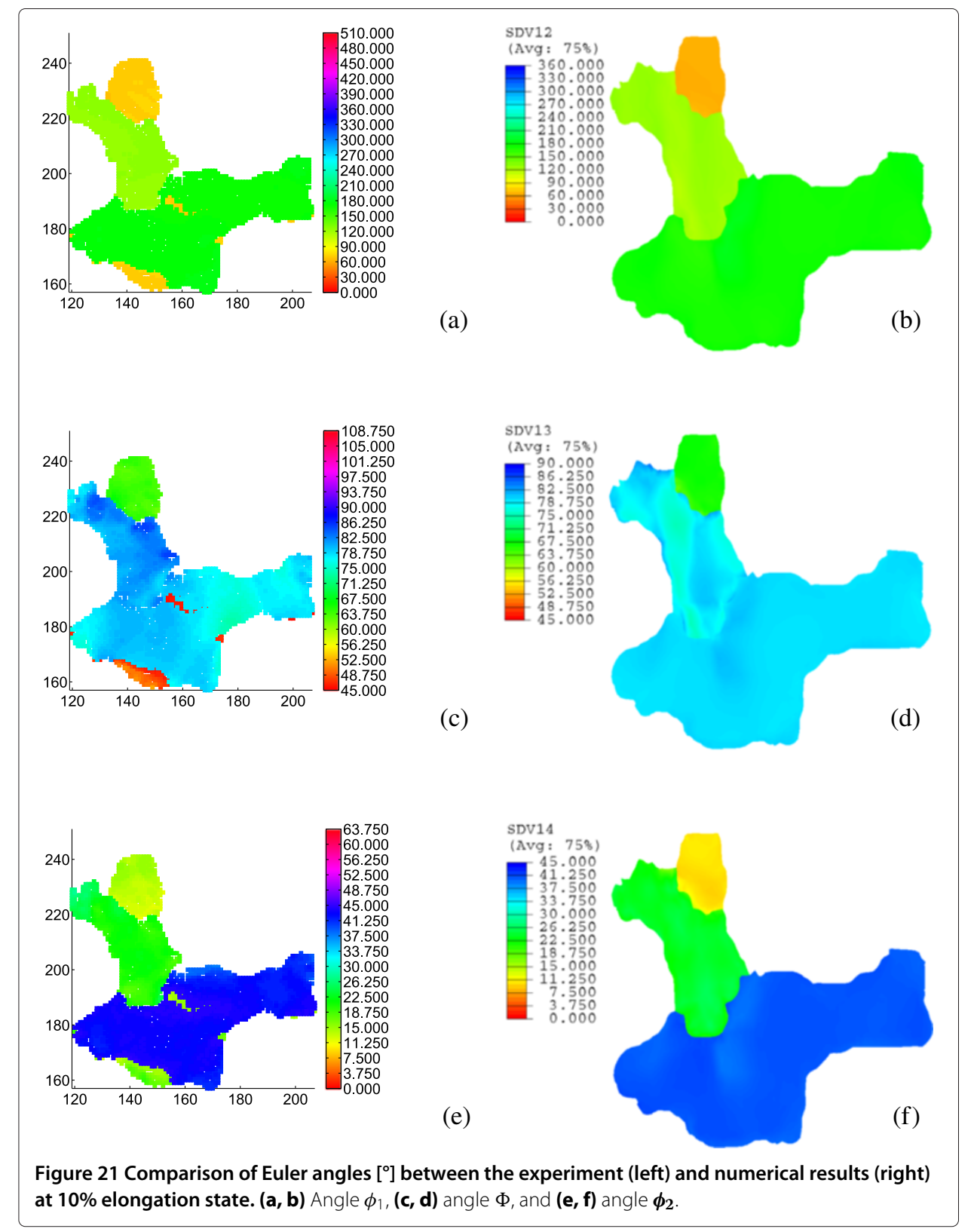

During the simulation, $\boldsymbol{Q}$ is extracted by the polar decomposition $\boldsymbol{F}_{e}=\boldsymbol{R}_{e} \boldsymbol{U}_{e}$, where $\boldsymbol{R}_{e}=\boldsymbol{Q}$ is the elastic rotation and $\boldsymbol{U}_{e}$ is the elastic stretch tensor. The computed reorientation is the minimum relative orientation distance between the initial and actual crystal orientation. By comparing the color distribution representing the values in the legend (Figure 24), the reorientations in the numerical simulations agree well with the experimental results. In addition, the computed reorientations of local grains \#345 and \#296 are in good agreement with the experiment. However, the computed reorientation of local grain \#357 is lower than in the experiment. This can probably be explained by neglecting the beneath grain interaction in the ND due to the lack of the experimental 2D EBSD data. 


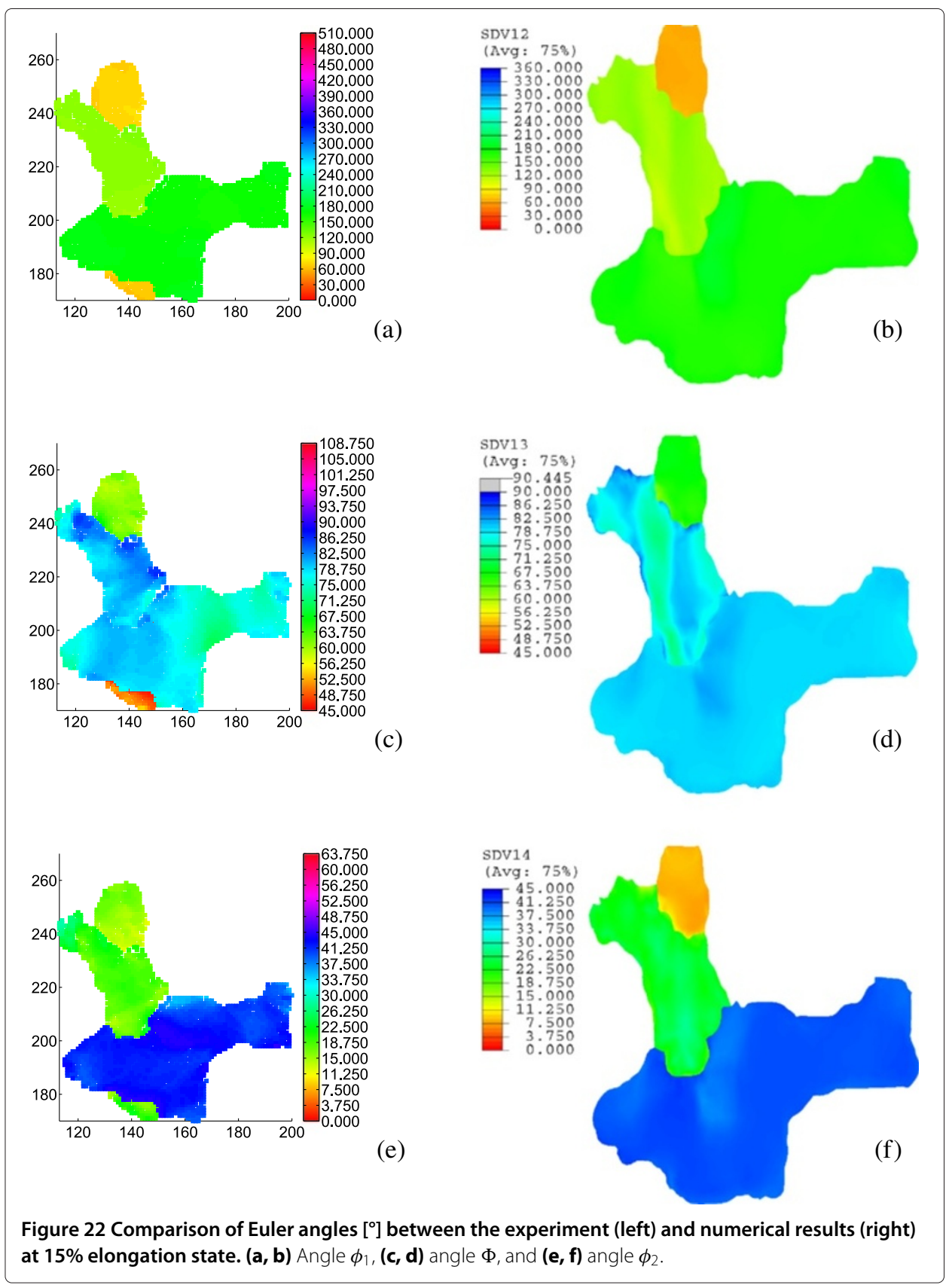

\section{Conclusions}

In the paper, a finite strain crystal plasticity model has been presented. The constitutive equations applied on the grain scale to model the elasto-viscoplastic behavior of BCC single crystals have been described in the context of large deformations. In particular, the crystal plasticity model is rate dependent and takes into account hardening effects on the microscale. The material parameters of the DC04 steel used in the crystal plasticity model were identified in the work of [25].

In addition, the experimental EBSD technique to obtain the microstructural information has been introduced. By using the EBSD measurement technique, a data set of a 


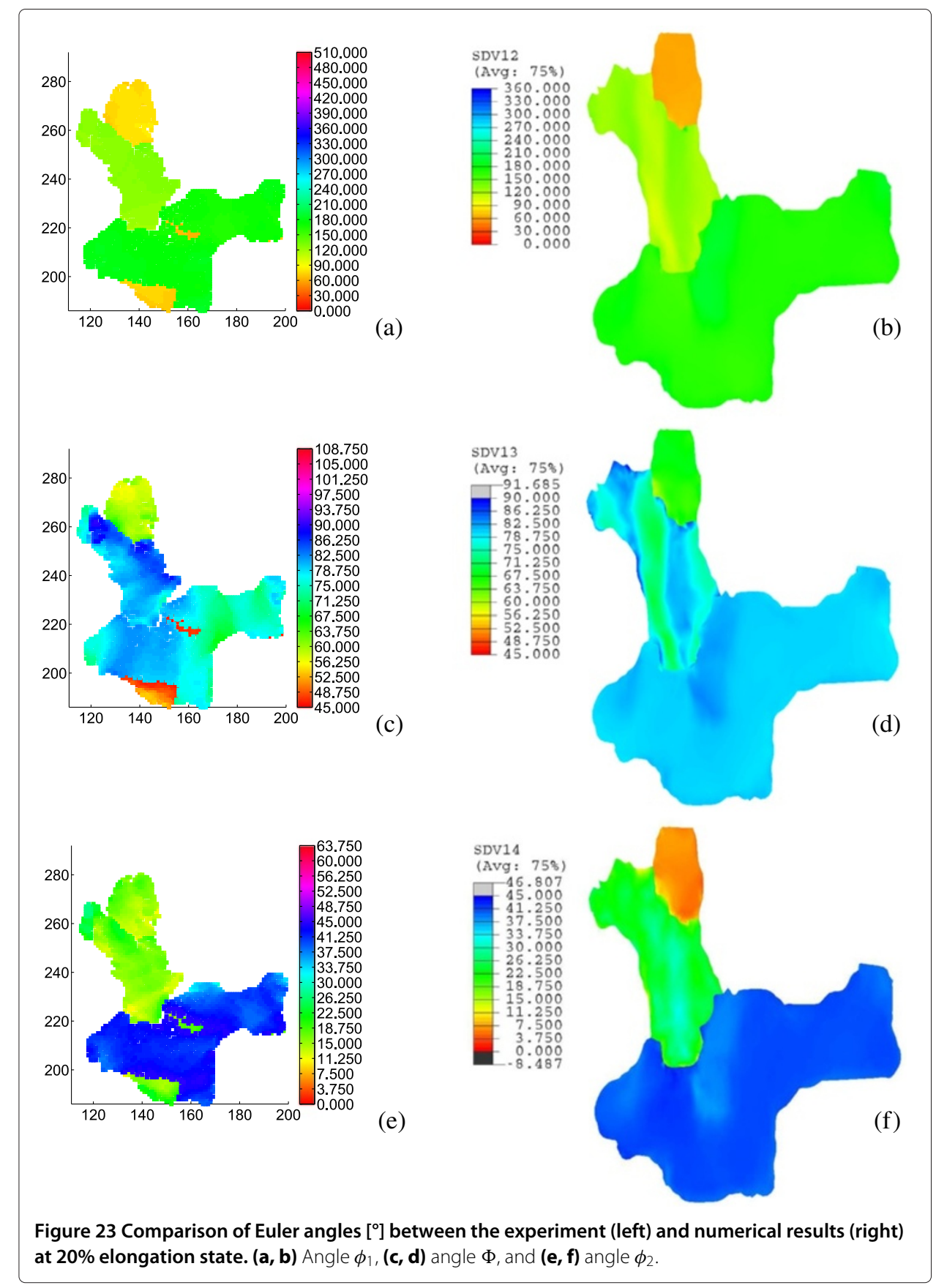

DC04 steel specimen in the cold formed and the heat-treated processes has been presented. In order to analyze and evaluate quantitatively the experimental texture data, a Matlab toolbox MTEX has been introduced and used. The MTEX allowed to import the different EBSD data formats and to obtain a 2D grain structure with corresponding crystal orientations. These EBSD data sets have been processed to identify the grain information and to obtain the clustered data.

A verification of the material model has been carried out. Based on EBSD data, the grain structure was modeled by a FE model. The FE mesh has been imported into the ABAQUS/CAE software for the performance of the grain scale simulation. This 


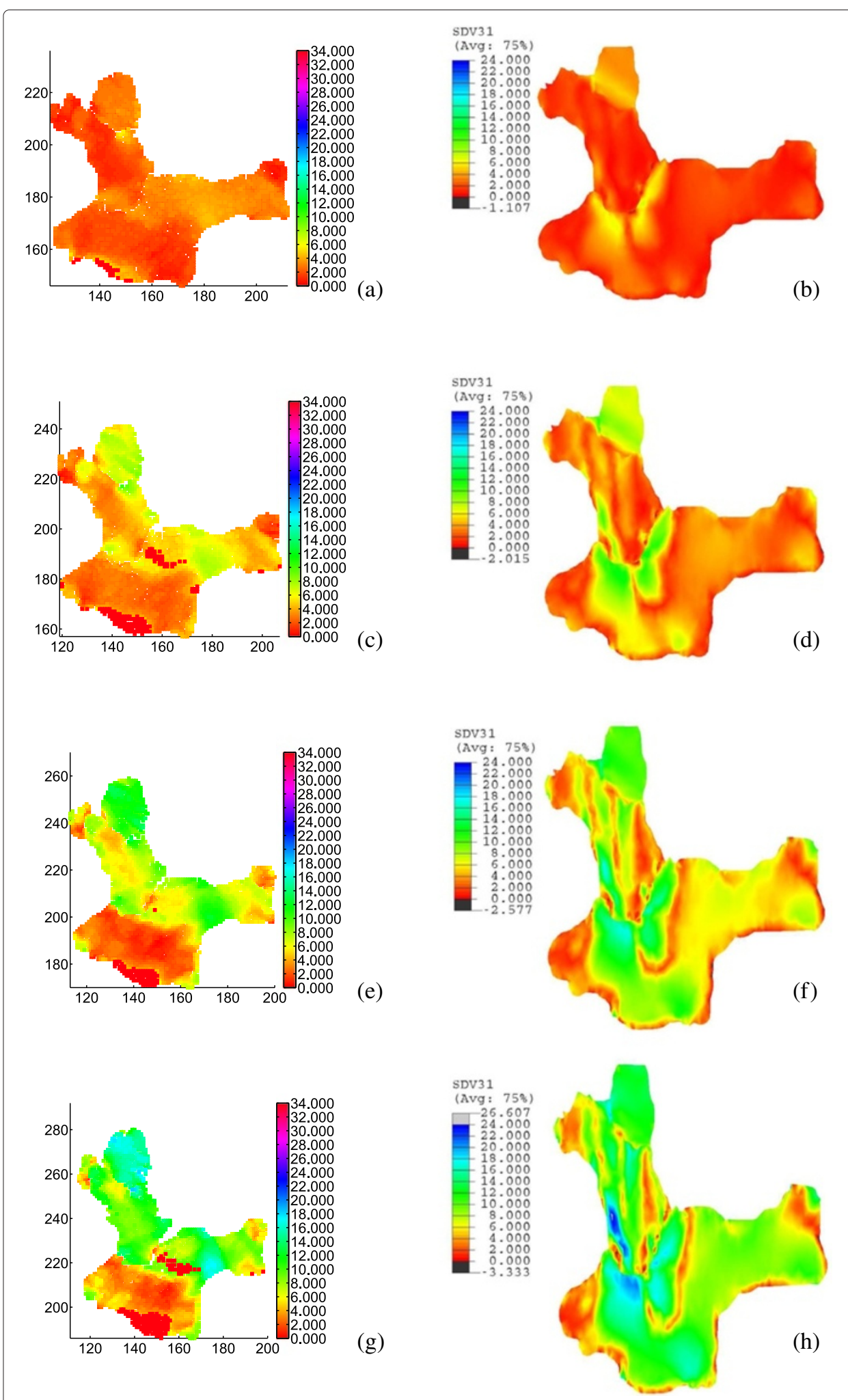

Figure 24 Comparison of reorientation $\left[^{\circ}\right]$ between the experiment (left) and numerical results (right) at different states. (a, b) 5\%, (c, d) 10\%, (e, f) 15\%, and (g, h) $20 \%$ elongation. 
FE simulation has been used for the identified DC04 steel material microparameters, and two families of the BCC slip systems were assumed to possibly act simultaneously, namely $\{110\}\langle 111\rangle+\{112\}\langle 111\rangle$. In addition, a procedure for mapping the initial grain orientations into the fundamental zone has been implemented. The micromechanical behavior was analyzed in terms of the evolution of grain orientations. The grain orientations and reorientations are compared to the experiment for a group of local grains. The numerical results of local grain reorientations seem to underestimate the heterogeneity compared to the experimental results. This issue can be probably explained by the neglect of the 3D microstructure and interaction of the beneath microstructure in the grain scale simulation. The influence of $3 \mathrm{D}$ interaction needs to be further investigated in further studies.

Competing interests

The author declares no competing interests.

\section{Acknowledgements}

The author acknowledges gratefully T. Böhlke (ITM, KIT) for his constructive contribution. The author also thanks S. Schreijäg and R. Mönig (IAM, KIT) for the supply of the EBSD data of DC04 steel in this research work.

Received: 31 December 2013 Accepted: 29 May 2014

Published: 15 July 2014

References

1. Bunge H (1993) Texture analysis in materials science. Culliver Verlag, Göttingen

2. Asaro RJ, Needleman A (1985) Texture development and strain hardening in rate dependent polycrystals. Acta Materialia 33: 923-953

3. Mathur K, Dawson P (1989) On modeling the development of crystallographic texture in bulk forming processes International Journal of Plasticity 5: 67-94

4. Bronkhorst CA, Kalidindi SR, Anand L (1992) Polycrystalline plasticity and the evolution of crystallographic texture in FCC metals. Philosophical Transactions of the Royal Society A 341: 443-477

5. Raabe D (1995) Simulation of rolling textures of b.c.c, metals considering grain interactions and crystallographic slip on 110, 112 and 123 planes. Materials Science and Engineering A 197: 31-37

6. Böhlke T (2000) Crystallographic texture evolution and elastic anisotropy: simulation, modeling, and application. Shaker Verlag $\mathrm{GmbH}$, Aachen

7. Böhlke T, Risy G, Bertram A (2005) A texture component model for anisotropic polycrystal plasticity. Computational Materials Science 32: 284-293

8. Böhlke T, Glüge R, Klöden B, Skrotzki W, Bertram A (2007) Finite element simulation of texture evolution and swift effect in NiAl under torsion. Modelling and Simulation in Materials Science and Engineering 15: 619-637

9. Roters F, Eisenlohr P, Hantcherli L, Tjahjanto DD, Bieler TR, Raabe D (2010) Overview of constitutive laws, kinematics, homogenization and multiscale methods in crystal plasticity finite-element modeling: theory, experiments, applications. Acta Materialia 58: 1152-1211

10. Jöchen K, Böhlke T (2013) Representative reduction of crystallographic orientation data. Journal of Applied Crystallography 46: 960-971

11. Lee EH (1969) Elastic-plastic deformation at finite strains. ASME Journal of Applied Mechanics 36: 1-6

12. Asaro RJ, Rice JR (1977) Strain localization in ductile single crystals. Journal of the Mechanics and Physics of Solids 25 : 309-338

13. Ting $T$ (1996) Anisotropy elasticity: theory and applications. Oxford University Press, New York/Oxford

14. Kocks U, Mecking H (2003) Physics and phenomenology of strain hardening: the FCC case. Progress in Materials Science 48: 171-273

15. Yalcinkaya T, Brekelmans WAM, Geers MGD (2008) BCC single crystal plasticity modeling and its experimental identification. Modelling and Simulation in Materials Science and Engineering 16(8): 085007

16. Paquin A, Berbenni S, Favier V, Lemoine X, Berveiller M (2001) Micromechanical modeling of the elastic-viscoplastic behavior of polycrystalline steels. International Journal of Plasticity 17: 1267-1302

17. Maitland T (2004) Electron backscattered diffraction: an EBSD system added to an SEM is a valuable new tool in the materials characterization. Advanced Materials and Processes 162(5): 34-36

18. Maitland T, Sitzman S (2006) Scanning microscopy for nanotechnology. Chapter 2: Electron backscatter diffraction (EBSD) technique and material characterization examples. Springer, New York, pp. 41-75

19. Schwartz AJ, Kumar M, Adams BL, Field DP (2009) Electron backscatter diffraction in materials science. 2nd edn. Springer, New York

20. Bunge H (1982) Texture analysis and materials science. Butterworths, London

21. Schreijäg S (2013) Microstructure and mechanical behavior of deep drawing DC04 steel at different length scales. PhD thesis, KIT Scientific Publishing, Karlsruhe

22. Hielscher R (2010) MTEX Quantitative Texture Analysis Software. http://code.google.com/p/mtex/. 1 Sept 2009

23. Bachmann F, Hielscher R, Schaeben H (2011) Grain detection from 2D and 3D EBSD data-specification of the MTEX algorithm. Ultramicroscopy 111: 1720-1733 
24. Simpleware (2000) Simpleware: converting 3D images into models. http://www.simpleware.com/. 15 Dec 2011

25. Phan VT (2012) Modeling the mesoscopic and macroscopic deformation behavior of the ferritic stainless steel DC04. PhD Thesis, Karlsruhe Institute of Technology, Karlsruhe

26. Gao X, Przybyla CP, Adams BL (2006) Methodology for recovering and analyzing two-point pair correlation functions in polycrystalline materials. Metallurgical and Materials Transactions A 37A: 2379-2387

27. Sudook AK, Ward LJ (2007) Elastic constants and internal friction of martensitic steel, ferritic-pearlitic steel, and alpha-iron. Materials Science and Engineering A 452-453: 633-639

doi:10.1186/s40540-014-0010-7

Cite this article as: Phan: Grain scale simulation of local deformation behavior. Asia Pacific Journal on Computational Engineering 2014 1:10

Submit your manuscript to a SpringerOpen ${ }^{\circ}$ journal and benefit from:

- Convenient online submission

- Rigorous peer review

- Immediate publication on acceptance

- Open access: articles freely available online

- High visibility within the field

- Retaining the copyright to your article

Submit your next manuscript at $\gg$ springeropen.com 